\title{
Resumen del mercado mundial del grafito y prospectiva de los yacimientos españoles
}

\author{
Javier Elez Villar y Francisco Javier Gonzalo Corral \\ SAMCA, Paseo de la Independencia, 21, 3. 50001 Zaragoza \\ jelezv@gmail.com; fgonzalo@samca.com
}

\begin{abstract}
RESUMEN
Este trabajo ofrece una visión general resumida de la situación actual del mercado internacional del grafito, focalizado tanto en los productos y usos finales de este elemento como en la evolución reciente de los precios y las expectativas de evolución a futuro de estos. También se realiza una síntesis de los proyectos de exploración y explotación más relevantes a nivel internacional, producciones anuales, reservas, etc. incidiendo en los principales problemas actuales de este mercado y su potencial evolución. Nos hemos centrado únicamente en el grafito natural, a pesar de que el sintético tiene un mayor campo de aplicación para algunos usos de alto valor añadido y ha sufrido un incremento muy importante de los precios estos últimos años debido a las medidas anticontaminación arbitradas por el gobierno chino.

Además, se ha realizado una investigación de las características de los yacimientos e indicios de grafito existentes en España, su situación actual y sus posibilidades de desarrollo futuro. Para ello se realiza una revisión de los indicios más relevantes conocidos y se aportan nuevos datos sobre algunos de los yacimientos más significativos.
\end{abstract}

Palabras clave: Baterías, España, Grafito, Yacimientos.

\section{Summary of the global graphite market and the prospectives for the spanish deposits}

\begin{abstract}
This study offers an overview of the current situation of the international graphite market, focused on the products and final uses of this metal as well as on the recent evolution of prices and expectations of its future evolution. There is also a synthesis of the most relevant international exploration and production projects, annual productions, reserves, etc. influencing the main current problems of this market and its potential evolution. We have focused only on natural graphite, although synthetic graphite has an important field of application for some uses of high added value and has experienced a very significant increase in prices in the recent years due to the anti-pollution measures arbitrated by the Chinese government.

In addition, we offer a review of the characteristics of the main deposits of graphite existing in Spain, their current situation and the possibilities for future development and we also provide new data about some of the most significant deposits.
\end{abstract}

Keywords: batteries, deposits, graphite, Spain.

\section{ABRIDGED ENGLISH VERSION}

Introduction

Broadly speaking, there is a significant expectation that in coming years the consumption, and therefore the demand for graphite will increase, mainly due to its use in the construction of battery anodes (USGS, 2017; Fig. 1). Therefore, despite the fact that in the last few years the total production of graphite remains relatively constant, prices have experienced a clearly ascending tendency (e.g. Roskil 2018c; Fig. 2 and 3), especially marked since 2006 (Industrial Minerals and Chinese Customs 2018). However, contrary to the most optimis- 
tic forecasts, only $10 \%$ of the graphite obtained is used today in the battery industry, with the metallurgy and refractory industries being the users that account for almost $70 \%$ of the consumption (Fig. 1).

In this paper, we offer a review of the situation of the current graphite market with an international scope, focusing mainly on 1) final uses of the graphite, 2) recent evolution of the prices and 3) relevant production and exploration projects. We have also considered it interesting to make a brief summary of the data regarding the main producing countries, describing the most relevant features and problems of each of them and their possible future evolution.

The ideas arising from the study of the international panorama motivated the SAMCA Group (Sociedad Anónima Minera Catalano Aragonesa) to promote research on graphite resources in Spain and a nationwide exploration campaign was carried out in 2016. From the data identified in the literature, a number of zones were selected:

- Guadamur (Toledo), the last of the graphite mines working in Spain which closed in the 1960s.

- Riaza-Sierra de Ayllon (Segovia), with historical descriptions of graphite associated to the Silurian Hot Shales (El Muyo and Becerril sites).

- Alto Tajo (near Orea, Guadalajara), where the analyses of the Silurian and Ordovician black shales made for the exploration of hydrocarbons indicated high carbon contents.

- Aroche and Cortegana (Huelva), here there is a relevant number of old graphite mining works (decades 1910-20).

\section{Current international market}

As far as geographical regions are concerned (Figs. 5 and 6 and Table 2), the main international mining projects are scattered throughout Europe (small projects), Asia (dominating China, India and North Korea), southern Africa (where Madagascar stands out), South America (only Brazil) and North America (Canada). It is also important to note that there are a large number of ongoing exploration projects (Table 2), both in the south-east of Africa, in the United States and especially Canada.

China is the largest producer of graphite in the world with an estimated total of 780,000 tons in 2017 and about $67 \%$ of world production, significantly more than the second world producer (India) with 150,000 $\mathrm{t}$ in 2017 (Fig. 7), and clearly with the capacity today to have an important influence on the international graphite market (Robinson et al., 2017)., China also produces $100 \%$ of the uncoated spherical graphite and about $90 \%$ of spherical graphite with coating. These derivatives represent a refinement, an increase in the purity of the product, and a change in the form of flakes to spheres of the aggregates of graphite so that the conductivity is improved and therefore it is the product required to make the anodes of lithium batteries.

Since the end of the 1970s, the price of graphite has risen from prices below USD $400 / t$ to the current ones (Fig. 2A). It was during 2005 (Industrial Minerals, 2010) when the prices rose significantly linking them with the current trends. Table 1 shows the prices of the most important grades and graphite products in the current market.

Published reports on the forecast of the evolution of graphite prices (e.g. Industrial Minerals and Chinese Customs 2018, Investing News Report 2018, Roskill 2018c) are optimistic. Roskill (2018b and c) indicates that since the end of 2017 and until the beginning of 2018 the strengthening of graphite prices is unmistakable, especially in the case of flakes which where the demand for the construction of battery anodes lies.

Amorphous graphite has many difficulties for its concentration in products with high percentages of carbon concentration and is used massively in the steel industry (Investing News Report, 2018). The flake type of graphite of varies in prices depending on the size of the flakes and this is the one that currently is of special interest since it is the one that is used as for battery anodes. As shown in Table 3, the more abundant types are amorphous and flake. The list of the graphite mines in the world with the largest capacity is also led by China (Table 4).

From a political point of view, in 2017 the European Union included graphite in the list of Critical Raw Materials for European industry.

\section{Results of the exploration of graphite in Spain}

After the analysis of the bibliographic data, a first field campaign was carried out in each of the selected areas to identify the units described in the literature and carry out specific mapping and sampling work. The obtained samples were analyzed for total organic content, (TOC) and X-ray diffraction (DRX) to characterize both the total carbon content and its degree of crystallinity. Mineral preparation and analysis were performed following the methods indicated by Mitchell (1993) and recommended by the British Geological Survey.

Regarding the exploration work carried out in Spain, it is worth pointing out that the target areas show a 
Javier Elez Villar y Francisco Javier Gonzalo Corral, 2019. Resumen del mercado mundial del... Boletín Geológico y Minero, 130 (1): $27-46$

modest exploratory interest, generally constrained by both the scarcity of the resource and environmental issues.

We have studied the old mines located in the Metamorphic Band of Aracena (Ossa Morena Zone, Quesada et al., 1994) La Niña (Figs. 12 and 13), Las Hormigas (Figs. 14 and 15) and San Carlos (Table 5), all of them few kilometres south of the town of Cortegana. There may also be other old mines in the town of Aroche, few kilometres to the west, of which there is hardly any documentary record or positive identification in the field.

The zone of the old mine of "La Niña" in the Metamorphic Band of Aracena was the most promising since mineralized gneissic units have a certain cartographic continuity over more than a hundred meters and the fixed carbon values were around $5-6 \%$ with peaks close to $10 \%$ (Fig. 12 and Table 6). However, in this area it is quite difficult to make a reliable prognosis of the underground lithological distribution given the complexity of the geological setting and further development of the exploratory work would involve geophysical surveying prospecting and/or a borehole campaign. The rest of the explored areas in the vicinity of Cortegana, Las Hormigas, Aroche area and the San Carlos mine showed less interest either due to the lack of the resource or to environmental issues (the old mine of Las Hormigas is within several areas of Environmental Protection).

In the Guadamur area, a vein, several hundred meters long, was mined up to 100 meters deep (Fig. 13), with an estimated thickness of two-three metres. Graphite mineralization oscillated between 3 and $20 \%$ of graphite showing a high degree of crystallization (Martín Méndez et al., 2015, 2017 and 2018). This mine facility obtained concentrates of up to $80 \%$ graphite. There are still outcrops of graphite in the meta-sediments of the Precambrian in small veins of only $10-20 \mathrm{~cm}$ thick and up to a metre long.

In the Sierra de Ayllón area, the analysis carried out offered relatively low fixed carbon contents, with Becerril being the highest (a sample taken from the old Becerril mine resulted in a $24.51 \%$ fixed carbon content attributable to graphite) in line with previous studies (Lasheras et al., 1995). Here, the graphite appears towards the top of some thickening and coarsening sequences in the black shales series of the Silurian (Fig. 14). It is usually found accumulated in levels of few centimeters and in the finest grained areas, these are relatively abundant and the sites have been identified in several locations (El Muyo, Becerril, Madriguera and Serracín; Puche Riart and Pedrazuela González, 1988, Lasheras et al., 1995).

In the case of the area in Orea-Alto Tajo, which is located mostly within a Natural Park, analytical results indicate very low contents of graphite (near $1 \%$ ). Here the mineralization was found associated to fault planes and genetic interpretation points to minor segregation of graphite from the black shales during Alpine faulting.

\section{Introducción}

En términos generales, existe una expectativa importante de que en los próximos años el consumo, y por tanto la demanda, de grafito se incremente debido fundamentalmente a su utilización en la construcción de ánodos de baterías eléctricas (USGS, 2017). En cuanto a los datos, desde el punto de vista internacional hay que destacar que a pesar de que en los últimos años la producción total de grafito se mantiene relativamente constante los precios tienen una clara tendencia ascendente (e.g. Roskil 2018c), especialmente marcada desde 2006 (Industrial Minerals and Chinese Customs 2018). Como se detallará más adelante, dentro de esta tendencia ascendente es importante resaltar que los precios del grafito en escamas, utilizado fundamentalmente para la fabricación de baterías, han sido los que más han crecido.

Es significativo que todo esto sucede en un contexto global en el que la demanda se incrementa muy ligeramente (USGS, 2017), ya que a pesar de las previsiones más optimistas solo el $10 \%$ del grafito beneficiado se utiliza a día de hoy en la industria de las baterías siendo los usos relativos a la metalurgia y refractarios los que ocupan casi el $70 \%$ de la producción (Industrial Minerals Graphite Maps, 2017). En este trabajo ofrecemos una revisión resumida de la situación del mercado actual del grafito a nivel internacional, incidiendo fundamentalmente en 1) usos finales del grafito, 2) evolución reciente de los precios y 3) proyectos de explotación y exploración más relevantes. También hemos considerado interesante hacer un breve resumen de los datos de los principales países productores, comentando las características y problemáticas más relevantes de cada uno de ellos y su posible evolución futura.

Las ideas surgidas del estudio del panorama internacional motivaron que el Grupo SAMCA (Sociedad Anónima Minera Catalano Aragonesa) decidiera impulsar la investigación de los recursos de Grafito en España y fundamentalmente a lo largo de 2016 se realizara una campaña de exploración a nivel nacional. En España, las primeras explotaciones de grafito de forma industrial se remontan al siglo XIX (Puche Riart and Pedrazuela González, 1988), sin embargo muchas de ellas fueron de tamaño muy pequeño para 
los estándares actuales. Del conjunto de indicios identificados en la literatura se eligieron una serie de zonas/yacimientos que a priori presentaban condiciones suficientes como para continuar una investigación más detallada. Estas zonas fueron:

- Guadamur (Toledo), la última mina de grafito que se explotó en España.

- Riaza-Sierra de Ayllon (Segovia), con descripciones históricas de grafito asociado a las pizarras negras del Silúrico (yacimientos de EI Muyo y Becerril).

- Alto Tajo (entorno de Orea, Guadalajara), en donde los análisis de las pizarras negras del Silúrico y Ordovícico realizados para la exploración de hidrocarburos indicaban contenidos altos en carbono.

- Aroche y Cortegana (Huelva), donde existían una serie de labores mineras antiguas relacionadas con la explotación de grafito (décadas de 1910-20).

Este trabajo resume los resultados de las labores de investigación realizadas, centrándose más en la zona de Aroche y Cortegana, en la que todos los indi- cios apuntaban a priori que era la más prometedora de todas las investigadas.

\section{Resumen del mercado actual del grafito}

\section{Destino del grafito}

Tal y como se observa en el gráfico de la Fig. 1, solo el $10 \%$ del grafito obtenido se utiliza a día de hoy (datos de 2017) en la realización de ánodos de baterías. Los usos más destacados corresponden a fundición y refractarios $(40 \%)$ y metalurgia $(28 \%)$. Incluso en la realización de lubricantes o en partes y componentes industriales se utilizan cantidades similares a las descritas para ánodos, sobre el $10 \%$. Por tanto y a pesar de lo anticipado, el grafito todavía no se destina mayoritariamente o en cantidades francamente significativas desde el punto de vista porcentual a la fabricación de ánodos de baterías de lon-Litio.

Como se detallará más adelante China es el mayor productor de grafito del mundo con un total estimado de 780000 toneladas en el año 2017 y cerca del $67 \%$

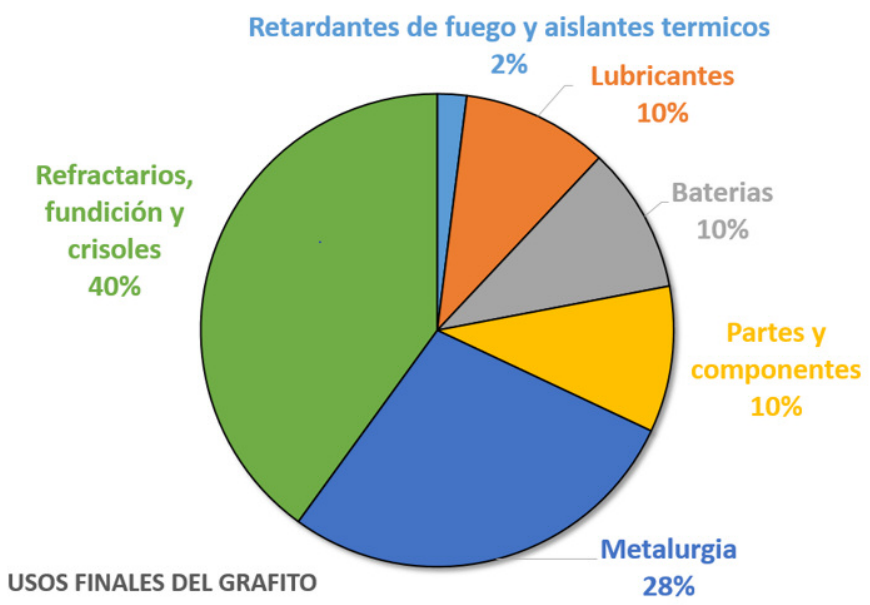

\begin{tabular}{|c|c|c|}
\hline Extracción $\quad$ Molid & zación Esferificación & Recubrimiento \\
\hline $\begin{array}{l}\text { Concentrado de láminas de grafito } \\
\text { El mas habitual en la industria del } \\
\text { Grafito. }\end{array}$ & $\begin{array}{l}\text { Grafito esférico sin recubrimiento } \\
\text { Procesado secundario con mas del } \\
99,95 \% \text { de } \mathrm{Cg} \text {. } \\
100 \% \text { producción China }\end{array}$ & $\begin{array}{l}\text { Grafito esférico con } \\
\text { recubrimiento } \\
\text { Procesado secundario con } \\
\text { mas del } 99,95 \% \text { de } \mathrm{Cg} \text {. } \\
90 \% \text { producción China }\end{array}$ \\
\hline
\end{tabular}

Figura 1. Usos finales del grafito en \% y proceso mina-ánodo. Destacar que la práctica totalidad de los productos de grafito con un cierto procesado (purificación, micronización, esferificación y/o recubrimiento) son realizados por China. Fuente: Industrial Minerals Graphite Maps, 2017.

Figure 1. Final uses of graphite in \% and mine-anode process. Note that practically all the graphite products with some processing (purification, micronization, spherification and/or coating) are made in China. Source: Industrial Minerals Graphite Maps, 2017. 
de la producción mundial, a una distancia muy importante del segundo productor mundial (India) con 150000 t en 2017, y claramente con la capacidad a día de hoy de influir de forma importante en el mercado internacional del grafito (Robinson et al., 2017). Sin embargo esto contrasta con los resultados de procesados de grafito (grafito esférico a partir de grafito en escamas) en los que China es el productor del $100 \%$ del grafito esférico sin recubrimiento y cerca del $90 \%$ de grafito esférico con recubrimiento (Fig. 1). Estos derivados suponen un refinamiento, incremento de la pureza del producto, y cambio de la forma de escamas a esferas de agregados de grafito de forma que se mejora la conductividad y por tanto es el producto requerido para la realización de ánodos de baterías de litio. Según Industrial Minerals esta actividad es muy costosa (http://www.indmin.com/Article/ 3238613/Spherical-graphite-how-is-it-made.html), tanto económica como medioambientalmente, habiendo sido incluso prohibida en varias ocasiones en China (Ghilotti, 2018) por su baja rentabilidad, encareciendo el producto final y haciéndola poco atractiva para compañías de otros países.

\section{Evolución de los precios}

Desde finales de la década de los 70 el precio del grafito ha subido desde precios inferiores a los 400 USD/t hasta los precios actuales (e.g. Fig. 2A). Es a a)

PRECIOS DEL GRAFITO EN USA

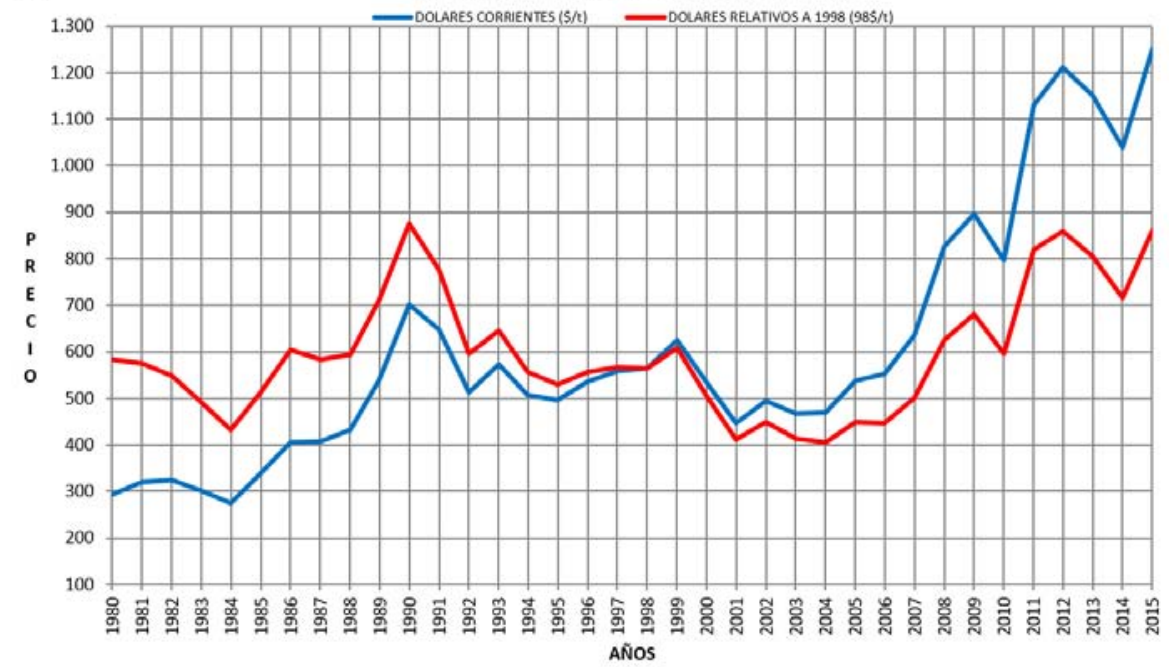

b)

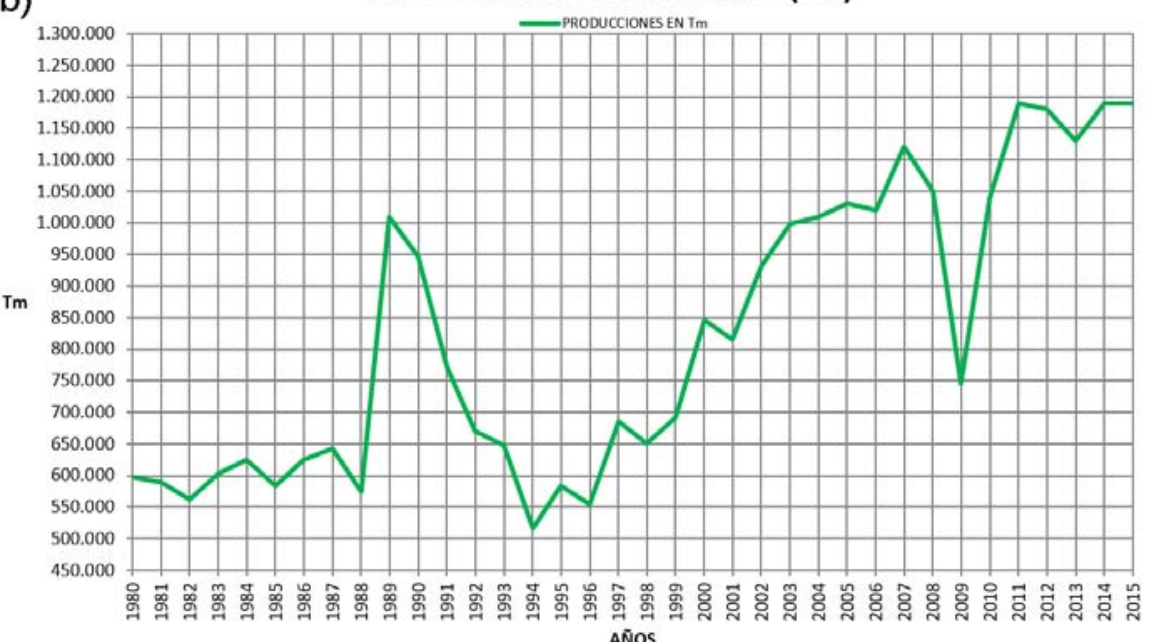

Figura 2. Evolución desde 1980 hasta 2015 de a) precios del grafito en los Estados Unidos y b) producción global de grafito, fuente USGS. Figure 2. Evolution from 1980 to 2015 of a) graphite prices in the United States and b) global production, source USGS. 


\begin{tabular}{|l|c|c|}
\hline & \multicolumn{2}{|c|}{ Precios en USD/t } \\
\hline Tipo de grafito & Low & High \\
\hline Graphite Flake, 85-87\% C, +100 Mesh -80 Mesh, FCL, CIF, Europe & 400 \\
\hline Graphite Flake, 90\% C, +100 Mesh -80 Mesh, CIF, Europe & 550 & 650 \\
\hline Graphite Flake, 90\% C, +80 Mesh, CIF, Europe & 600 \\
\hline Graphite Flake, 90\% C, -100 Mesh, FLC, CIF, Europe & 500 \\
\hline Graphite Flake, 94-97\% C, +100 Mesh -80 Mesh, FLC, CIF, Europe & 950 & 550 \\
\hline Graphite Flake, 94-97\% C, +80 Mesh, FLC, CIF, Europe & 1000 \\
\hline Graphite Flake, 94-97\% C, -100 Mesh, FLC, CIF, Europe & 790 \\
\hline Graphite Amorphous, 80-85\% C, -200 Mesh, FCL, CIF, China to Europe & 400 \\
\hline
\end{tabular}

Tabla 1. Precios del grafito a 7 de Junio de 2018. Fuente: Industrial Minerals.

Table 1. Graphite prices as of June 7, 2018. Source: Industrial Minerals.

partir de 2005 (Industrial Minerals, 2010) cuando se producen los repuntes de precios más importantes que enlazan con las tendencias actuales. Esto ha ido unido a un incremento también generalizado de la producción en el mismo intervalo temporal (Fig. 2B). En laTabla 1 se especifican los precios de las diversas calidades y productos de grafito más importantes en el mercado actual.

Sin embargo en los últimos dos años no se han producido modificaciones significativas en los pre- cios de estos productos salvo en el caso del grafito laminar $94-97 \%$ de $\mathrm{C}$ que es el que se destina a fabricar el grafito esférico (ver Fig. 3). Esto probablemente se deba a que la producción ha crecido solo ligeramente y a que la demanda solo crece significativamente en el grafito para la fabricación de baterías, con la industria del acero lastrada por el bajo crecimiento de ésta en China en los últimos años que ha bajado en su demanda (Investing News Report, 2018, Roskill, 2018b y c).

\section{Graphite Flake, 94-97\% C, +80 Mesh, FCL,CIF, Europe (USD/tonne)}

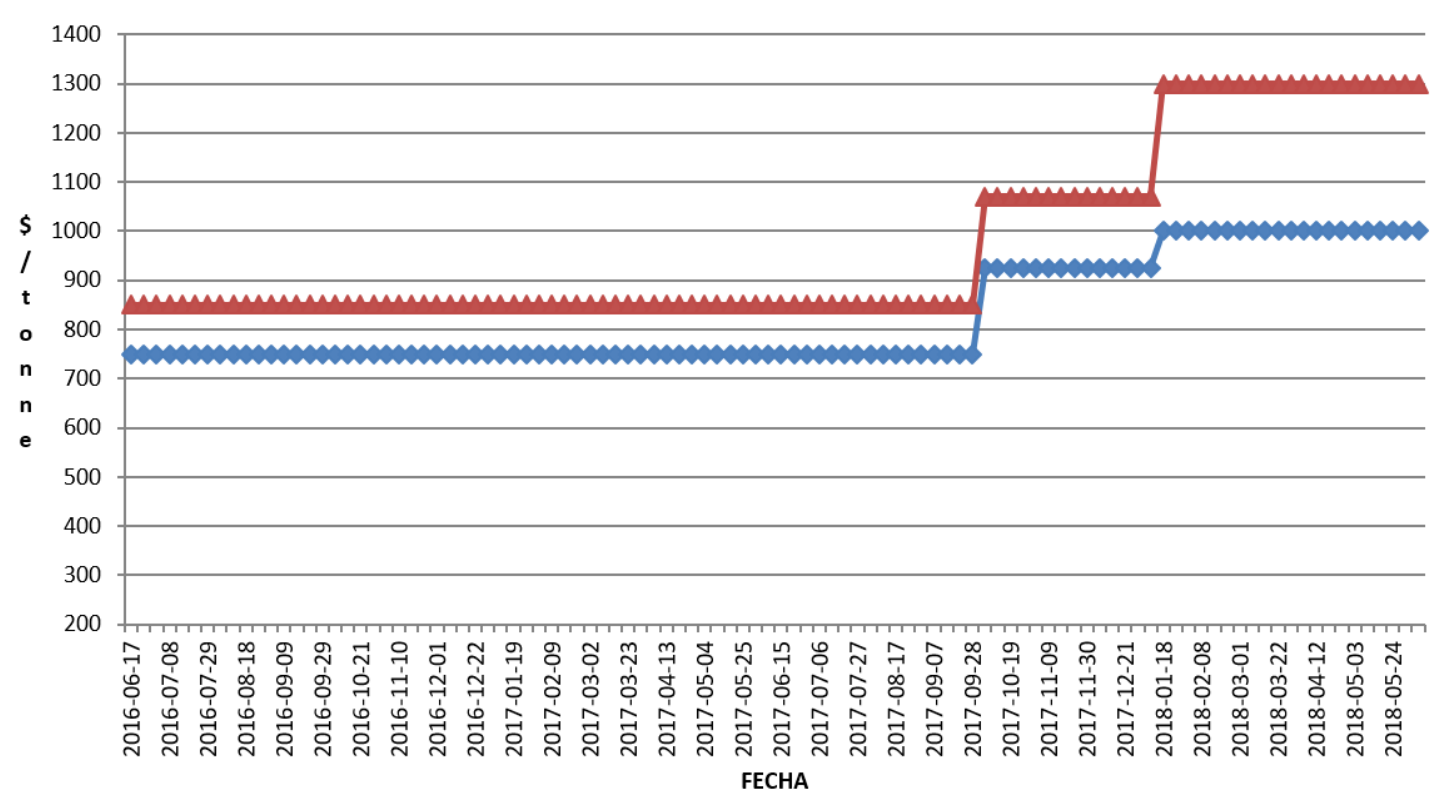

Figura 3. Evolución en los últimos años del precio del grafito laminar 94-97\% de C + 80 mesh, datos tomados de Industrial Minerals. Figure 3. Recent evolution in the price of laminar graphite $94-97 \%$ of $C+80$ mesh, data from Industrial Minerals. 
En cualquier caso, los informes publicados sobre la previsión a futuro de la evolución de los precios del grafito (e.g. Industrial Minerals and Chinese Customs 2018, Investing News Report 2018, Roskill 2018c,) son optimistas. Roskill (2018b y c) indican que desde finales de 2017 y hasta principios de 2018 se evidencia el fortalecimiento de los precios de grafito, sobre todo en el de escamas que es el que se demanda para la construcción de ánodos de baterías, en esta línea se puede observar en la Fig. 4 como las exportaciones de grafito esférico chinas crecen en los últimos tres años. Concretamente, en el segundo cuatrimestre de 2018 el precio del "94-97\% C flake" es un 35-45\% superior a su valor a mitad del año pasado (Roskill 2018c citando a Industrial Minerals). Los mismos autores indican que ha subido de forma significativa el precio de los electrodos de grafito sintético a lo largo de todo 2017 y que la demanda de grafito por parte de la industria siderúrgica se está recuperando en estos momentos, haciendo que las previsiones de consumo a futuro de este mineral se incrementen también por esta vía.

\section{Proyectos internacionales de explotación y explora- ción más relevantes}

Por regiones geográficas (Figs. 6, 7 y 8), las explotaciones se encuentran repartidas por Europa (explotaciones pequeñas), Asía (dominando China, India y Corea del Norte), sur de África (donde destaca Madagascar) y Sudamérica (únicamente Brasil) y
Norteamérica (fundamentalmente Canadá). Algunos de estos proyectos se comentarán brevemente en el siguiente apartado dedicado a los principales países productores. Mucho más en detalle, Robinson et al. (2017) ofrece una serie de datos sobre localización, grado, tonelaje, etc. para más de cien depósitos y distritos activos (o no) y de cobertura global.

Es de destacar un numeroso conjunto de proyectos de exploración en marcha (Tabla 2), tanto en el sureste Africano como en Estados Unidos y sobre todo Canadá. Aquí, junto a las dos explotaciones mayores en funcionamiento, se están desarrollando nueve proyectos de exploración de tamaño relevante en distintos estados de evolución.

\section{Resumen de los datos relativos a los principales paí- ses productores}

Según el Servicio Geológico Americano (USGS), la producción de grafito a lo largo de los últimos años no se ha modificado de forma significativa (Fig. 2B). Encabezan la lista de países productores China, India, Brasil, y Canadá, con Turquía como país con la mayor cantidad de reservas (Fig.9). Estados Unidos, uno de los mayores consumidores no posee recursos ni reservas de este mineral. Para la mayor parte de países las producciones calculadas por el World Mineral Data 2018 (Reichel et al., 2018, y publicado por el Federal Ministry of Sustainability and Tourims de Austria) son ligeramente menores a las del USGS, por ejemplo Brasil e India para los que estiman pro-

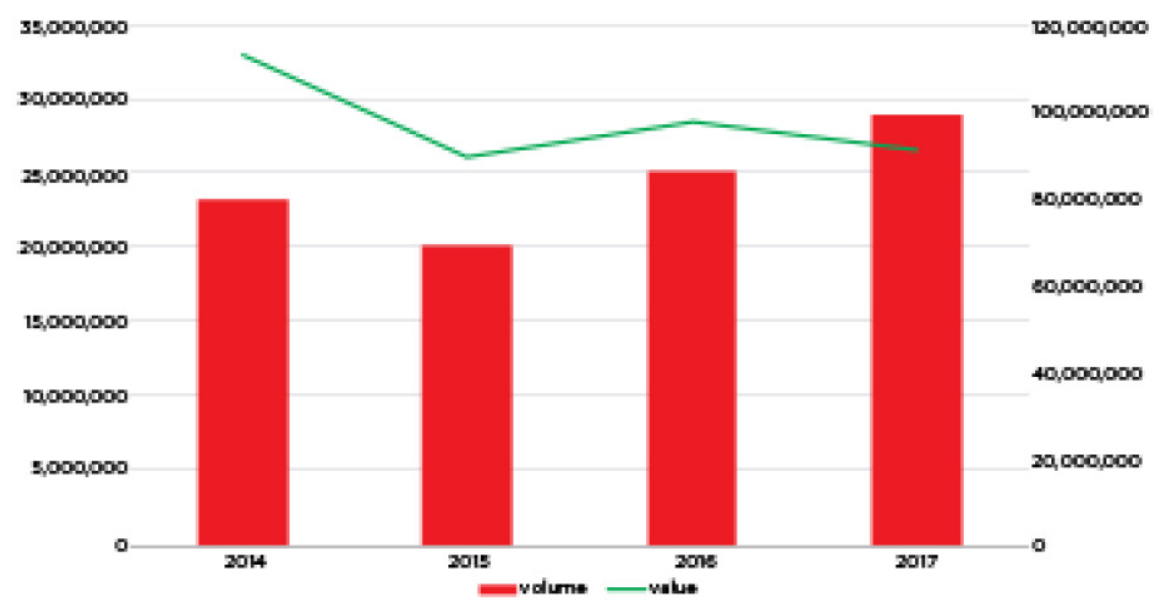

Figura 4. Exportaciones chinas de grafito esférico (2014-2017, precio en \$ y volumen en kg; tomado de Industrial Minerals and Chinese Customs, 2018).

Figure 4. Chinese exports of spherical graphite (2014-2017 price in \$ and volume in kg; from Industrial Minerals and Chinese Customs, 2018). 


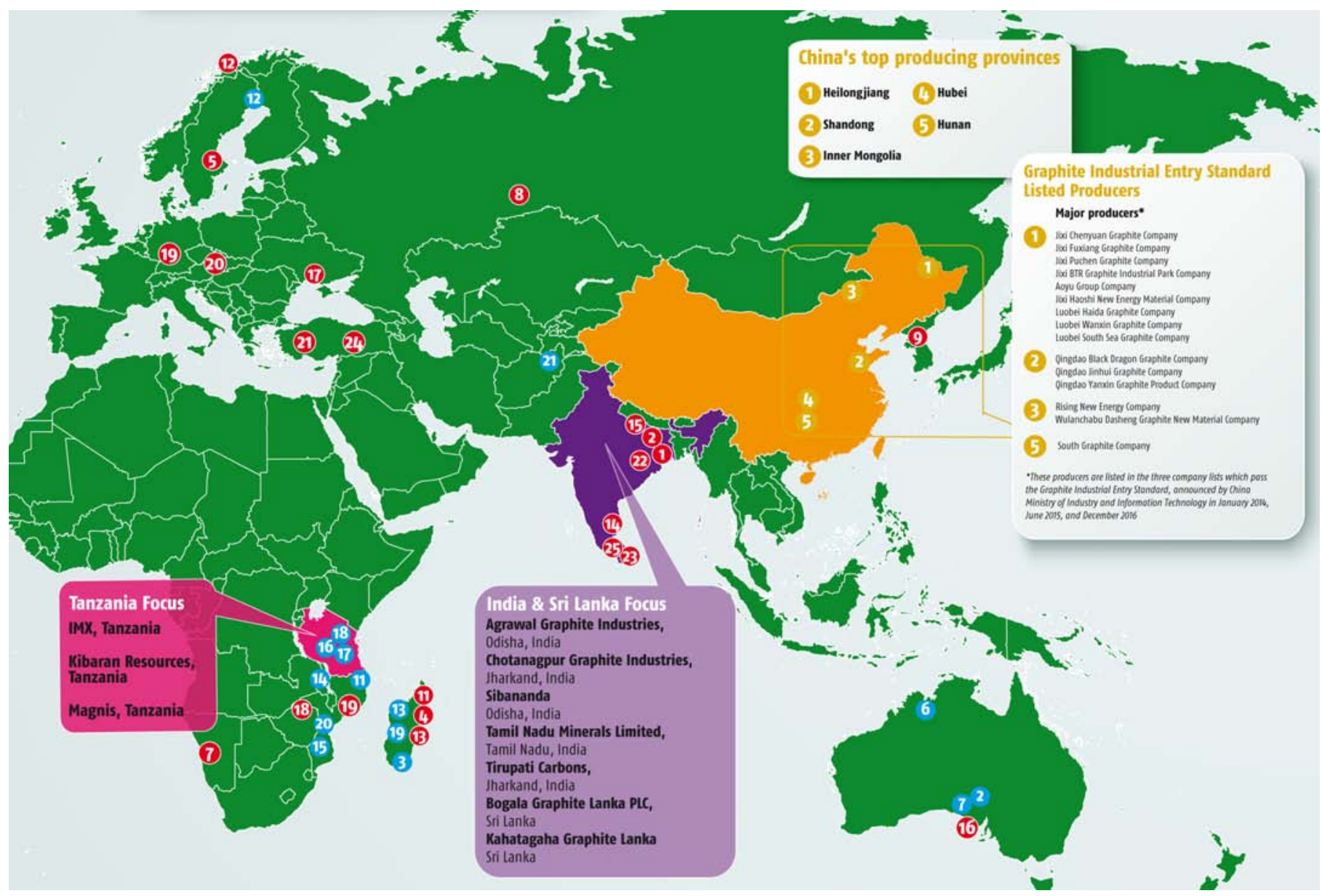

Figura 5. Mapa de localización de los yacimientos en explotación (rojo) y los proyectos de exploración en estado avanzado (azul), en las regiones de Europa, Asia, África y Oceanía. Tomado de Industrial Mineral Graphite World Maps (2017). Listado de proyectos en Tabla 2.

Figure 5. Map with location of major producers (red) and major exploration projects (blue), regions of Europe, Asia, Africa and Oceania; from Industrial Mineral Graphite World Maps (2017). List of projects in Table 2.

ducciones de 23000 y 27000 toneladas menos respectivamente. Sin embargo, la producción estimada para China por el Servicio Geológico Británico (Brown et al., 2018) duplica largamente (1800000 toneladas) las dos anteriores aun cuando para otros países arroja cifras fácilmente comparables.

La producción mundial de grafito se reparte entre grafito amorfo, escamas (flake) y venas (vein). Como se observa en el gráfico de la Tabla 3, los más abundantes son los de tipo amorfo y en escamas. El grafito de tipo amorfo presenta muchas dificultades para su concentración en productos con porcentajes de concentración de carbono alto y se utiliza de forma masiva en la industria siderúrgica (Investing News Report, 2018). El grafito de tipo escama varía en precios dependiendo del tamaño de las escamas y es el que presenta en estos momentos un interés especial ya que es el que, tras su procesado (esferificación y recubrimiento) se utiliza como ánodo de baterías.
A continuación se aportan algunos de los datos más relevantes tanto de los primeros productores (China, India, Brasil y Canadá) como del país con las mayores reservas (Turquía).

China es el mayor productor de grafito del mundo con una producción en 2017 cercana a los 780000 toneladas (Fig. 7). Con respecto al grafito es difícil precisar las minas más relevantes en este país (e.g. Industrial Minerals Graphite World Maps, 2017). Aparentemente una parte importante de la producción se realizaba (al menos hasta 2011) en minas pequeñas, algunas con componentes prácticamente artesanales. Sin embargo, a partir de 2011 se ejecutan una serie de planes impulsados por el estado y financiados por grandes empresas productoras para centralizar y modernizar todo el proceso minero.

Por ejemplo, South Graphite Co pretendía en 2011 que sus minas en la zona minera de Lu He Tai (https://www.diytrade.com/china/manufactu- 


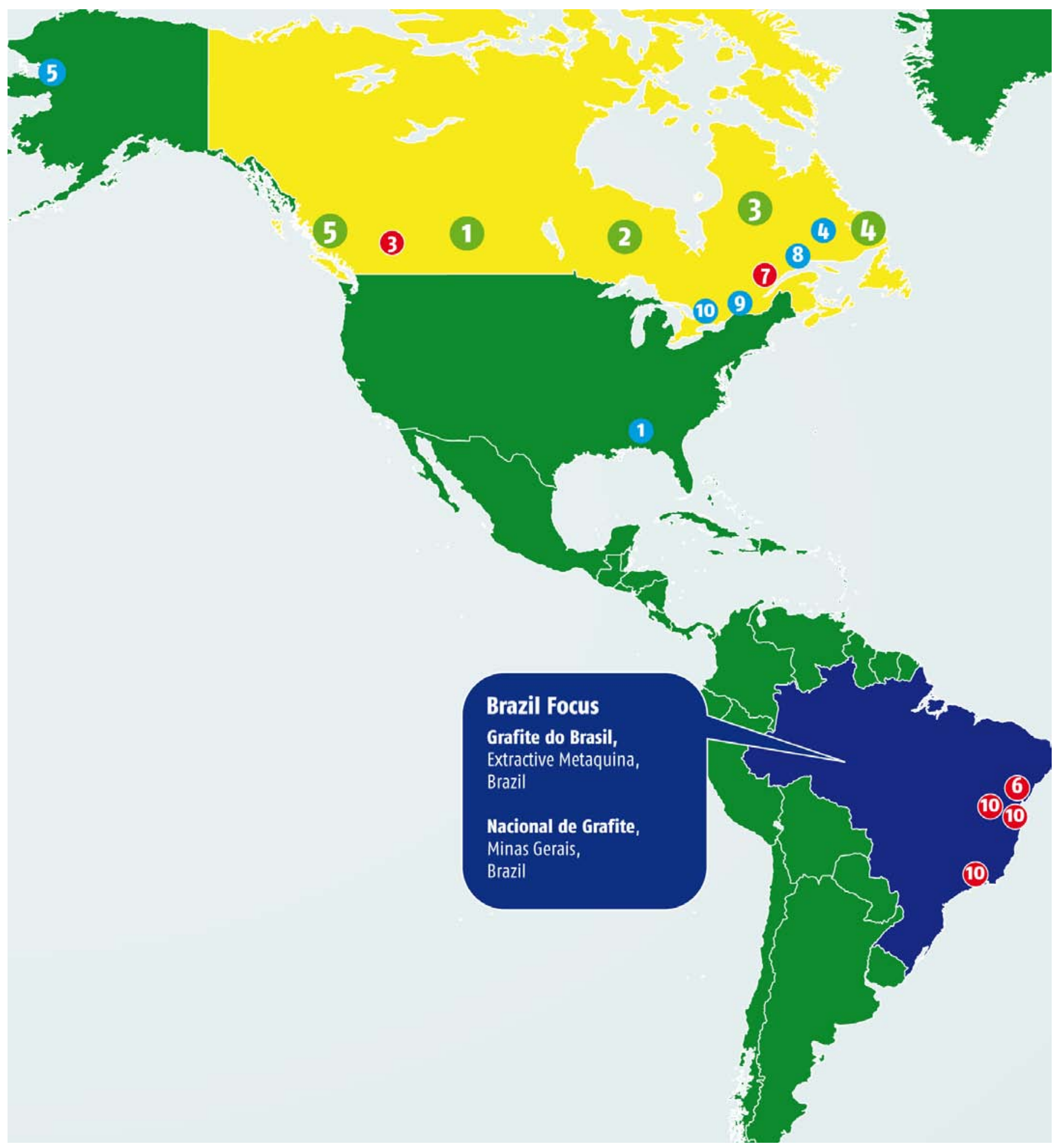

Figura 6. Mapa de localización de los yacimientos en explotación (rojo) y los proyectos de exploración (azul) en estado avanzado en la región de América; tomado de Industrial Mineral Graphite World Maps (2017). Listado de proyectos en Tabla 2. Los puntos verdes en Canadá sitúan las zonas que están siendo exploradas en estos momentos, totalizando más de 30 proyectos de exploración individuales e involucrando a una veintena de empresas; 1 Saskatchewan, 2 Ontario, 3 Quebec, 4 New Brunswick y 5 British Columbia.

Figure 6. Map with location of major producers (red) and major exploration projects (blue), region of America; from Industrial Mineral Graphite World Maps (2017). List of projects in Table 2. Green dots in Canada locate the areas currently under exploration, with more than 30 individual exploration projects involving some 20 companies; 1 Saskatchewan, 2 Ontario, 3 Quebec, 4 New Brunswick y 5 British Columbia. 


\begin{tabular}{|c|c|}
\hline Proyectos de Explotación & Proyectos de exploración \\
\hline $\begin{array}{l}1 \text { Agrawal Graphite Industries } \\
\text { Odisha, India }\end{array}$ & $\begin{array}{l}1 \text { Alabama Graphite Alabama, } \\
\text { USA }\end{array}$ \\
\hline $\begin{array}{lcc}2 & \text { Chotanagpur Graphite } \\
\text { Industries Jharkhand, India }\end{array}$ & 2 Archer Exploration Australia \\
\hline $\begin{array}{l}3 \text { Eagle Graphite Corp. Nelson, } \\
\text { Canada }\end{array}$ & \begin{tabular}{|ll}
3 & Energizer \\
Madagascar
\end{tabular} \\
\hline $\begin{array}{l}4 \text { Etablissements Gallois SA } \\
\text { Toamasina, Madagascar }\end{array}$ & $\begin{array}{ll} & \text { Focus Graphite } \text { Quebec, } \\
\text { Canada }\end{array}$ \\
\hline $\begin{array}{l}5 \text { Leading Edge Materials } \\
\text { Gävleborg County, Sweden }\end{array}$ & 5 Graphite One Alaska, USA \\
\hline $\begin{array}{l}6 \text { Grafite do Brasil Extractive } \\
\text { Metaquina, Brazil }\end{array}$ & 6 Hexagon Resources Australia \\
\hline $\begin{array}{l}7 \text { Imerys Graphite \& Carbon Lac- } \\
\text { des-Îles, Canada,Otjiwarongo, } \\
\text { Namibia }\end{array}$ & 7 Lincoln Minerals Australia \\
\hline $\begin{array}{l}8 \text { JSC Uralgraphite Taiginsky, } \\
\text { Russia }\end{array}$ & $\begin{array}{l}8 \text { Mason Graphite Quebec, } \\
\text { Canada }\end{array}$ \\
\hline $\begin{array}{l}9 \text { Myeong ji Mining } \mathrm{Co} / \\
\text { Jeongchon, North Korea }\end{array}$ & $\begin{array}{l}9 \text { Northern Graphite Ontario, } \\
\text { Canada }\end{array}$ \\
\hline $\begin{array}{l}10 \text { Nacional de Grafite Minas } \\
\text { Gerais, Brazil }\end{array}$ & $\begin{array}{l}10 \text { Ontario Graphite Ontario, } \\
\text { Canada }\end{array}$ \\
\hline $\begin{array}{l}11 \text { Power Stand Development } \\
\text { Atsinanana, Madagascar }\end{array}$ & 11 Syrah Resources Mozambique \\
\hline $\begin{array}{l}12 \text { Skaland Graphite Traelen, } \\
\text { Norway }\end{array}$ & 12 Talga Resources Suecia \\
\hline $\begin{array}{l}13 \text { Stratmin Global Resources } \\
\text { Loharano, Madagascar }\end{array}$ & $\begin{array}{l}13 \text { National Graphite Corp. } \\
\text { Madagascar }\end{array}$ \\
\hline $\begin{array}{l}14 \text { Tamil Nadu Minerals Limited } \\
\text { Tamil Nadu, India }\end{array}$ & 14 Sovereign Malawi \\
\hline $\begin{array}{l}15 \text { Tirupati Carbons Jharkhand, } \\
\text { India }\end{array}$ & $\begin{array}{l}15 \text { Graphit Kropfmühl (AMG-GK) } \\
\text { Nipacue, Mozambique }\end{array}$ \\
\hline $\begin{array}{l}16 \text { Valence Industries Uley, } \\
\text { Australia }\end{array}$ & 16 IMX Tanzania \\
\hline $\begin{array}{lll}17 & \text { Zavalyevsky } & \text { Graphite } \\
\text { Complex Zavalye, Ukraine }\end{array}$ & 17 Kibaran Resources Tanzania \\
\hline $\begin{array}{l}18 \text { Zimbabwe German Graphite } \\
\text { Mines Karoi, Zimbabwe }\end{array}$ & 18 Magnis Tanzania \\
\hline $\begin{array}{l}19 \text { Graphit Kropfmühl (AMG-GK) } \\
\text { Kropfmühl mine, Germany }\end{array}$ & 19 DNI Metals Madagascar \\
\hline $\begin{array}{l}20 \text { Grafitbergbau Kaiserberg } \\
\text { Loeben, Austria }\end{array}$ & $\begin{array}{l}20 \text { Damodar Ferro Lda Maputo, } \\
\text { Mozambique }\end{array}$ \\
\hline $\begin{array}{l}21 \text { Oysu Graphite Mining Co. } \\
\text { Kütahya,Turkey }\end{array}$ & $\begin{array}{l}1 \text { Various deposits (Flake) } \\
\text { Afganistán }\end{array}$ \\
\hline 22 Sibananda, Rayaganda, India & \\
\hline $\begin{array}{l}23 \text { Bogala Graphite Lanka, } \\
\text { Bogala Mines, Sri Lanka }\end{array}$ & \\
\hline $\begin{array}{l}24 \text { Karabacak Mining, Kütahya, } \\
\text { Turkey }\end{array}$ & \\
\hline $\begin{array}{l}24 \text { Kahatagaha Graphite Lanka, } \\
\text { Sri Lanka }\end{array}$ & \\
\hline
\end{tabular}

Tabla 2. Listado de proyectos de explotación y explotación más relevantes a nivel internacional. Tomado de Industrial Minerals Graphite World Maps, 2017.

Table 2. List of the major international producers and exploration projects; from Industrial Minerals Graphite World Maps, 2017.

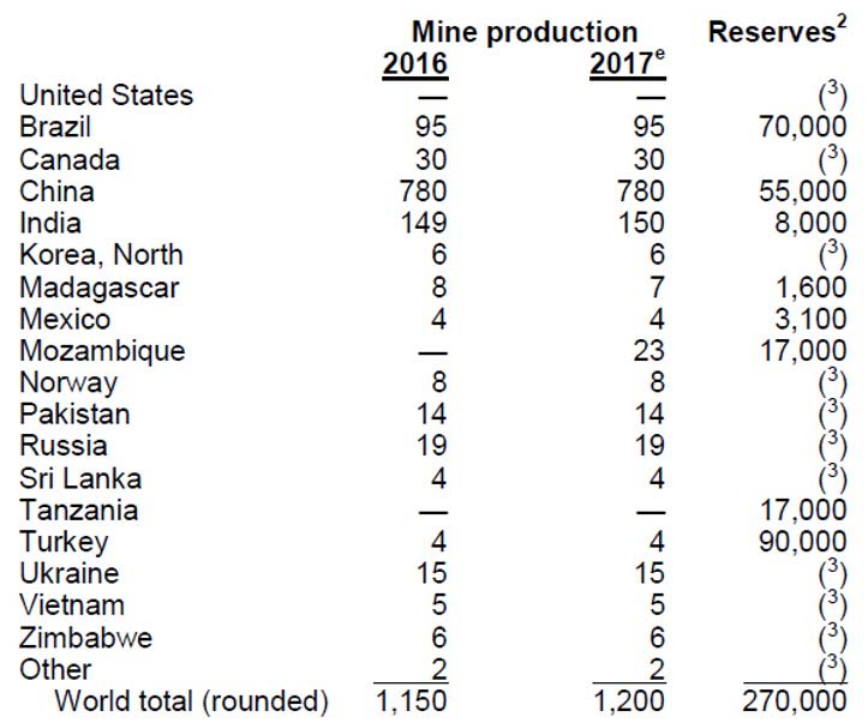

\section{En miles de toneladas}

Figura 7. Principales países productores en los años 2016 y 2017 y reservas; fuente USGS año 2017. Datos en miles de toneladas.

Figure 7. Main producing countries 2016 and 2017 and reserves; source USGS year 2017. Data in thousands of tons.

rer/1618632/main/South_Graphite_Co_Ltd.html, muy cerca de la ciudad de Chenzhou) produjeran unas 2.07 millones de toneladas (Mt) de grafito amorfo al año realizando para ello una inversión de unos 3000 millones de yuans (algo más de 400 millones de euros). Esta zona minera tiene unas dimensiones de $45 \mathrm{Km} 2$ en una banda alargada de más de $20 \mathrm{~km}$ de longitud. La zona también tiene explotaciones de carbón asociadas. Las reservas probadas son de 34.07 Mt de grafito (aproximadamente el $72 \%$ de las reservas probadas de grafito amorfo en China). El producto final que obtienen tras su tratamiento contiene sobre un $80 \%$ de carbono con picos de hasta el $92 \%$ de máximo.

Para el grafito en escamas, la práctica totalidad de la producción se sitúa en las provincias de Heilongjiang y Shandong, la primera es donde hay

\begin{tabular}{|l|l|l|}
\hline \multicolumn{3}{|c|}{ Producción mundial de grafito por tipo } \\
\hline En escamas (laminar) & Amorfo & En venas \\
\hline $73 \%$ & $26 \%$ & $1 \%$ \\
\hline
\end{tabular}

Tabla 3. Producción mundial de grafito por tipo. Tomado de Industrial Minerals Graphite Maps, 2017.

Table 3. Global production of graphite by type; from Industrial Minerals Graphite Maps, 2017. 
una mayor producción y la segunda es el centro más importante de tratamiento y procesado. En general, es complicado analizar la situación de este país debido a la carencia de datos públicos disponibles. En cuanto a la producción, según Industrial Minerals (2011) los datos de producción de minas/zonas mineras de grafito en toneladas por año apuntaban a las minas/zonas mineras chinas como las más productivas (Tabla 4).

En cualquier caso, todos estos datos son difícilmente comprobables ya que al menos en inglés o español no hay informes o estadísticas oficiales disponibles y no se ha encontrado ninguna publicación que indique de manera clara cuales son las producciones, minas, zonas mineras, empresas, normativas, etc. que se encuentran en el mercado chino.

India es el segundo productor de grafito del mundo con una producción en 2017 de 170000 toneladas. Según datos del Servicio Geológico de la India recogidos en el Indian Minerals Yearbook 2013 (Indian Bureau of Mines, 2015) y dedicado al grafito, India tiene unas reservas de $10.75 \mathrm{Mt}$ de grafito, con unos recursos disponibles de $158.02 \mathrm{Mt}$. Del conjunto, las minas de los estados de Jammu y Kashmir producen el $37 \%$ del total y las de Arunachal Pradesh el $43 \%$. Para 2013 había 16 minas operativas en ese país, cinco de las cuales producían el $84 \%$ del total del metal. En general los recursos de grafito que contienen $>40 \%$ de carbono fijo ascienden $1.11 \mathrm{Mt}$ y los que

\begin{tabular}{|l|l|l|}
\hline \multicolumn{1}{|c|}{ Compañía } & \multicolumn{1}{|c|}{ Localización } & Capacidad (tpa) \\
\hline $\begin{array}{l}\text { Jixi Liumao Graphite } \\
\text { Resource }\end{array}$ & Heilongjiang, China & 90000 \\
\hline $\begin{array}{l}\text { Heilongjiang Aoyu } \\
\text { Graphite Group }\end{array}$ & Heilongjiang, China & 80000 \\
\hline $\begin{array}{l}\text { Qingdao Haida Graphite } \\
\text { Co. }\end{array}$ & Shandong, China & 75000 \\
\hline $\begin{array}{l}\text { Chenzhou Luteng } \\
\text { Crystalline Graphite }\end{array}$ & Hunan, China & 70000 \\
\hline Nacional de Grafite & Bahía, Brasil & 70000 \\
\hline $\begin{array}{l}\text { Karaback Metal \& } \\
\text { Mining }\end{array}$ & Turquia & 50000 \\
\hline $\begin{array}{l}\text { Qingdao Hensen } \\
\text { Graphite }\end{array}$ & Shandong, China & 38000 \\
\hline LubeiYxiang Graphite & China & 30000 \\
\hline Extractive Metaquina & Bahía, Brasil & 30000 \\
\hline $\begin{array}{l}\text { Grafitbergbau } \\
\text { Kaiserberg }\end{array}$ & Austria & 30000 \\
\hline
\end{tabular}

Tabla 4. Capacidad de las minas más importantes de grafito del mundo, en toneladas de grafito por año. Fuente IM.

Table 4. Capacity of the most important graphite mines in the world, in tons of graphite per year. Source IM. presentan entre el 10 y el $40 \%$ a $22.69 \mathrm{Mt}$. Parece que lo habitual en estas explotaciones es obtener un todo uno con aproximadamente un $10 \%$ de carbono fijo que se trata múltiples veces para obtener concentrados comerciales. En el mencionado informe se cita alguna explotación en la que se explotan todo en uno con aprox. $5 \%$ de carbono fijo pero siempre mezclando con material de leyes más altas.

Brasil es el tercer productor de grafito del mundo con una producción en 2017 en torno a las 95.000 toneladas. Este país presenta dos zonas mineras principales que se reparten entre los estados de Minas Gerais y Bahía. Las compañías responsables de las explotaciones son Nacional de Grafite, Grafite do Brasil y Grafita MG. Los datos que a continuación se comentan están tomados principalmente del informe de Lobato (2009), denominado Brasil Perfil da Grafita, que incluye numerosos datos sobre la producción, geología y marco económico de las explotaciones de grafito en Brasil. Este documento técnico fue realizado por una empresa consultora a petición del Servicio Geológico Brasileño y con el objeto de poner al día los planes de desarrollo mineros en el intervalo temporal 2010-2030.

Nacional de Grafite, con las minas situadas en el estado de Minas Gerais, localidades de Itapecerica, Pedra Azul y Salto da Divisa, produce cerca de 70000 $t$ año. La planta más moderna, situada en Itapecerica tiene una capacidad instalada máxima de $56.000 \mathrm{t}$. En 2004 la producción en Itapecerica fue de $10530 \mathrm{t}$, en Pedra Azul 35041 t y en Salto da Divisa 8736 t. La mineralización que se explota presenta contenidos de entre el 6 y $8 \%$ de Carbono fijo y los concentrados que obtienen van del 89 al $94 \%$.

Grafite do Brasil (Extractive Metalquimica; FASA) es propietaria de la mina a cielo abierto Maiquinique, localizada en la región sur del estado de Bahia. Tiene una de las mayores reservas de grafito con unas 70 $\mathrm{Mt}$ de metal, unos $800 \mathrm{Mt}$ de todo en uno con entre el $10-12 \%$ de carbono fijo. La producción es de 54000 t/año. Obtienen un concentrado de hasta el $98 \%$ de carbono.

Grafita MG, con minas en los municipios de Serra Azul y Mateus Leme (estado de Minas Gerais) produjo 17307 t en el año 2004. La concentración de grafito en el todo uno llega al $14 \%$.

Canadá presenta una producción anual en 2017 de grafito de 30000 t. La explotación más importante de grafito situada en Canadá es la mina de Lac-des-lles, perteneciente aTimcal (Imerys SA). Esta se encuentra a $150 \mathrm{~km}$ al noroeste de Montreal y ocupa unos 40 $\mathrm{km}^{2}$. El grafito se explota de una secuencia metamórfica de mármoles, gneises, cuarcita y anfibolitas en las que las estructuras controlan la mineralización. 
Aparentemente, esta explotación tiene unas reservas limitadas que exponen a un periodo de vida corto a esta explotación (http://www.lecourant.ca/articles/230-fin-du-gisement-de-graphite-dans-5ans.html). Esto podría ser la razón del importante número de proyectos de exploración existentes en estos momentos en Canadá.

Turquía presenta una más que notable variabilidad en la producción de grafito, con años cercanos a $30000 \mathrm{t}$ y otros con resultados inferiores a las $5000 \mathrm{t}$. La mina que la compañía Karaback Metal and Mining tiene en la localidad de Kutahya es una de las que más capacidad máxima presenta a nivel mundial de grafito amorfo, con una producción futura cercana a las 50000 t/año. En este caso también parece que el mercado se compone de un numeroso grupo de pequeños productores, lo que explicaría las fuertes variaciones en la producción.

Mozambique, justo en el inicio de 2018 se ha publicado el éxito del proyecto Montepuez (ASX BAT), que ha pasado de fase de exploración a producción. Se estima una producción de 50000 t/año de un concentrado de escamas con un $96.7 \%$ de grafito y que su producción pueda llegar a ser de 100000 t/año en 2022. La expectativa es que ya en 2019 pueda empezar a exportar (https://www.mining-technology.com/ projects/montepuez-graphite-project/). Esto situaría a Mozambique como uno de los mayores productores a nivel mundial con reservas estimadas para unos 30 años.

Otros países de África con proyectos importantes de producción de grafito. Además de los ya citados, existen algunos proyectos más en avanzado estado de desarrollo en Tanzania, Madagascar y Namibia donde Imerys tiene un proyecto de investigación junto con la compañía Gecko Graphite Namibia (Industrial Minerals Graphite Maps, 2017, Ghilotti, 2018, Roskill, 2018a, etc).

Dentro de la Unión Europea e incluyendo Noruega (país participante en el Espacio Económico Europeo) existen cuatro proyectos principales en explotación y uno de características importantes en fase de exploración (Talga Resources en Suecia), ver Industrial Minerals Graphite Maps (2017). De los proyectos en producción, el proyecto sueco de Gävleborg (Leading Edge Materials) produjo unas 295 toneladas de grafito en 2015 pero nada en 2016, el noruego (Traelen, por Skaland Graphite) 8000 toneladas en 2017 (datos del USGS 2017), el proyecto en Alemania (Kropfmühl, AMG-GK) produjo 502 toneladas en 2016 y el austriaco en Loeben (Grafitbergbau Kaiserberg) presentó una producción de 150 toneladas en 2016, datos tomados de Reichl et al. (2018).

Desde el punto de vista político, la Unión Europea incluyó en 2017 al grafito en la lista de materias primas críticas para la industria europea (http://ec.europa.eu/growth/sectors/raw-materials/specificinterest/critical_en), siendo además un elemento fundamental dentro de la iniciativa de la Comisión Europea denominada Alianza Europea de las Baterías.

\section{Resultados de la exploración de grafito en España}

Como se comentaba en la introducción, en España las primeras explotaciones industriales de grafito que se citan en documentos históricos datan finales del siglo XIX en la zona de la Sierra de Ayllón (Puche Riart and Pedrazuela González, 1988) y de principios del siglo XX en Aroche y Cortegana (Lasheras et al., 1995). Por ejemplo, en Huelva se sucedieron una multitud de pequeños registros mineros asociados a los indicios de grafito, hasta 66, entre 1916 y 1920 . El interés por el grafito surge durante la Segunda Guerra Mundial y se debe a su utilización en la fabricación de crisoles de fundición. En la mayoría de las concesiones sólo se realizaron pequeñas labores de exploración (catas, pozos de poca profundidad) que no tuvieron éxito y fueron rápidamente abandonadas. La última mina activa de grafito española se cerró en 1963 y se localizaba en Guadamur (Toledo). Con estos antecedentes se realizó una búsqueda sistemática de la información histórica existente, fundamentalmente perteneciente al IGME (e.g. Lasheras et al., 1995) y de las varias publicaciones científicas e informes técnicos desarrollados en las últimas décadas al respecto (ver Crespo et al., 2004 y las referencias allí citadas). De esta forma, la organización del plan de investigación desarrollado consistió inicialmente en una búsqueda bibliográfica y documental de la información geológica y minera existente, incluyendo analíticas, cartografías, etc. Una vez analizada esta información se eligen para continuar la investigación las cuatro zonas mencionadas (Guadamur, Riaza-Sierra de Ayllón, Alto Tajo, Aroche y Cortegana) ya que son las que más y mejores indicios ofrecen a priori (ver Fig. 8A).

Tras el análisis de los datos bibliográficos se realizó una primera campaña de campo en cada una de las zonas seleccionadas para identificar las unidades descritas en la literatura, mapearlas según el caso, y tomar muestras de las litologías de interés. A estas muestras se les realizaron análisis de TOC (Total Organic Content) y DRX (Difracción de Rayos X) para caracterizar tanto el contenido total en carbono como su grado de cristalinidad. Desde el punto de vista metodológico y para la caracterización mineral se siguieron las recomendaciones recogidas en el infor- 
me de Mitchell (1993) del Servicio Geológico Británico, similares a las utilizadas por Luque et al. (1993). A partir de los resultados de los análisis obtenidos, se realizaron nuevas campañas de campo. A continuación se destacan las características geológicas y geoquímicas principales de cada una de estas zonas.

\section{Zona de Aroche y Cortegana}

Localizadas en la Banda Metamórfica de Aracena (zona de Ossa Morena; Quesada et al., 1994) se han estudiado las antiguas minas de La Niña (Fig. 8), Las Hormigas, Aroche y San Carlos. Del conjunto e labores de investigación antiguas solo se desarrolló en su momento un número pequeño de minas. Aparte de las estudiadas, también debía haber otro conjunto minero activo en el mismo pueblo de Aroche, del que apenas queda registro documental ni identificación positiva en campo.

A día de hoy, los afloramientos existentes son de calidad baja a muy baja (Fig. 9B), la zona está muy vegetada y además sobre prácticamente todas las litologías se encuentra de forma ubicua un potente suelo, que en ocasiones va acompañado por una zona de fuerte alteración con potencias métricas. Las mineralizaciones de grafito se encuentran principalmente a techo de formaciones Gneísicas (Gneis de Fuente del Oro) y fuertemente deformadas (Fernández Rodríguez et al., 1996). Aparecen en cuerpos lenticulares, a veces estratoligados, con buzamientos altos, no continuos y mezclados con otras litologías. El grafito (Fig. 9C) suele aparecer desde

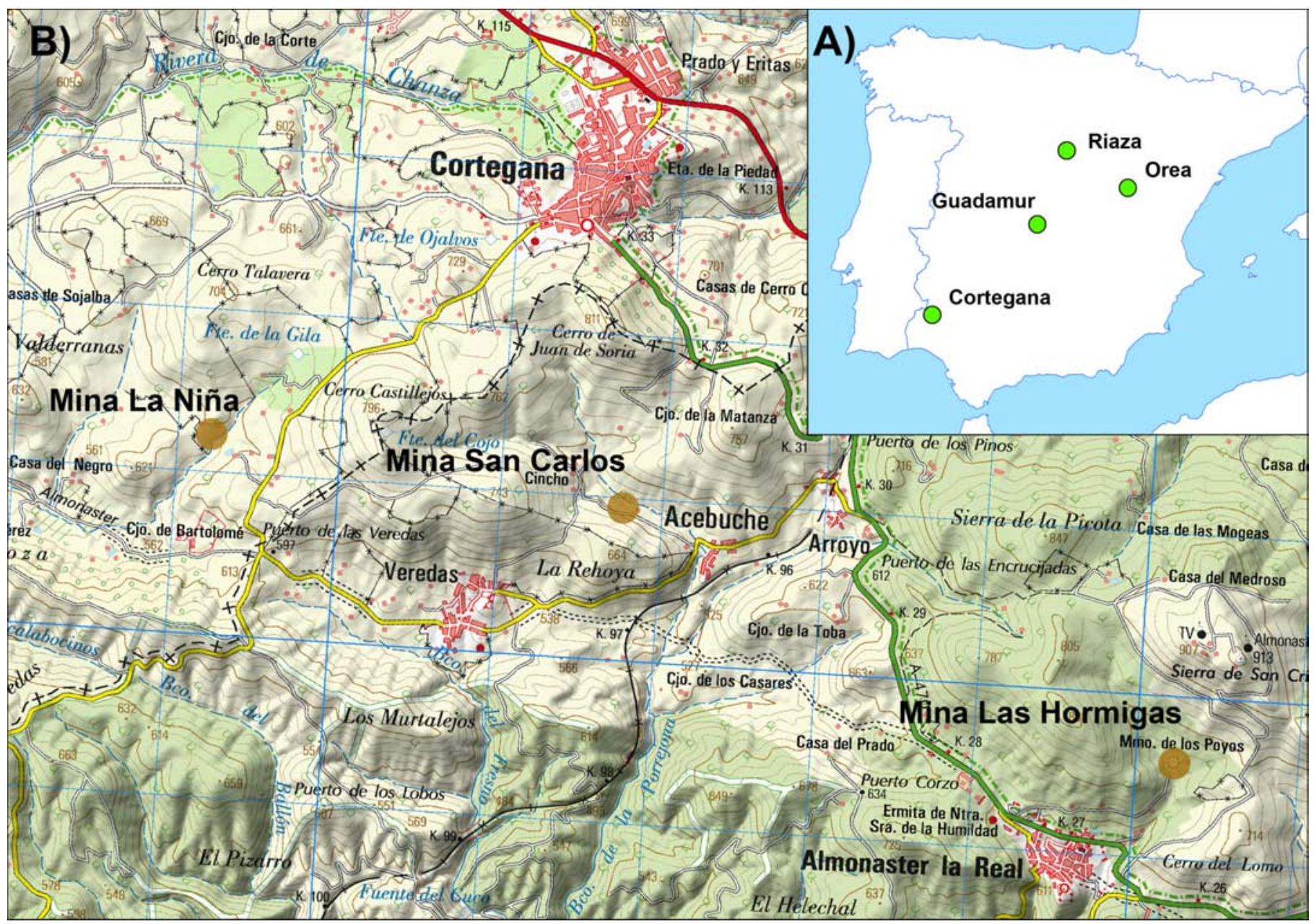

Figura 8. A) localización de las áreas estudiadas. B) localización de las antiguas zonas mineras estudiadas en el entorno de Cortegana en la Banda Metamórfica de Aracena.

Figure 8. A) location of the studied areas. B) location of the old mining areas in the vicinity of Cortegana in the Banda Metamórfica de Aracena. 
niveles de pocos milímetros hasta masivos de hasta 50-70 m de ancho (zonas de La Niña o Las Hormigas). En esta zona, los resultados de analíticas realizadas (Rodas et al., 2000) indicaban unos contenidos totales cercanos al $80-90 \%$ en peso de grafito, una vez flotados. En este mismo trabajo se indica que los cristales de grafito poseen morfología laminar o escamosa, y un tamaño comprendido entre 0.25 y $1 \mathrm{~mm}$. Estos aparecen formando agregados cristalinos o rellenando los espacios intergranulares.

Genéticamente, los autores que han trabajado en esta zona (e.g. Rodas et al., 2000, Crespo et al., 2004) interpretan que las mineralizaciones de grafito se ori- ginaron por metamorfismo regional de alta temperatura y baja presión que afectó a la Banda Metamórfica de Aracena durante la Orogenia Varisca. La materia orgánica dispersa en los sedimentos originales evolucionó hasta formar grafito de elevada cristalinidad a temperaturas estimadas del orden de $800{ }^{\circ} \mathrm{C}$.

En el entorno de Aroche no se Ilegaron a poder identificar las antiguas labores mineras de grafito, interpretamos que hoy en día destruidas o enterradas bajo nuevas edificaciones. En la antigua mina de "Las Hormigas" (Fig. 10) los resultados de una única muestra indican valores algo superiores al $13 \%$ de carbono atribuible a grafito (Fig. 11), el más alto del
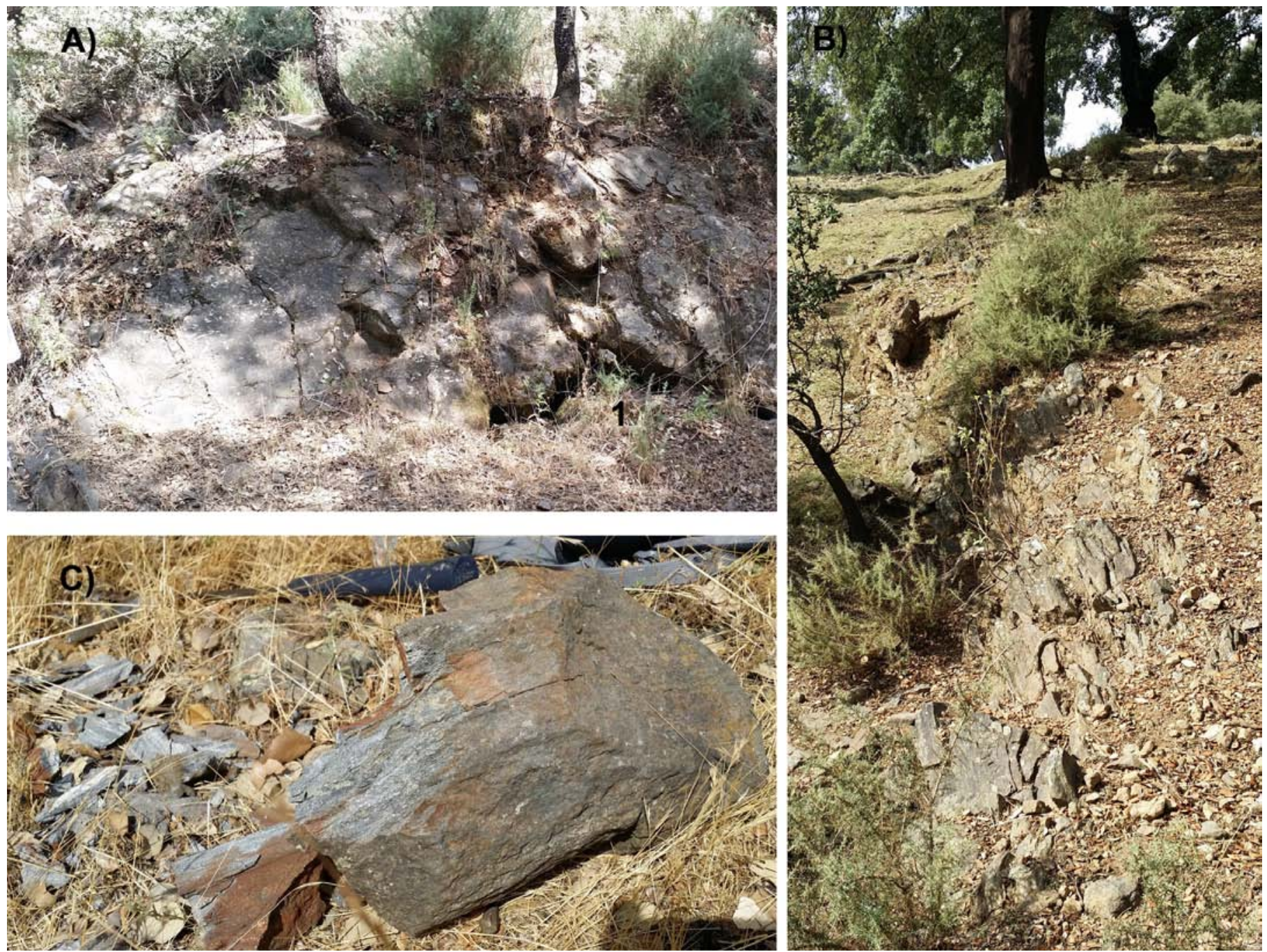

Figura 9. A) Aspecto actual de la entrada (1) a la antigua mina de La Niña y aspecto general del gneis mineralizado con grafito. B) aspecto general del gneis de Fuente del Oro aflorante, presenta buzamientos altos y está mineralizado con grafito. C) foto de detalle de los gneises con grafito. Las tres fotos están tomadas en el entorno de la mina La Niña.

Figure 9. A) Current appearance of the entrance (1) to the old La Niña mine and general appearance of the gneiss mineralized with graphite. $B)$ gneiss of Fuente del Oro outcrop showing high dips and mineralized with graphite. C) detail of the gneisses with graphite. The three photos are taken in the vicinity of the old La Niña mine. 


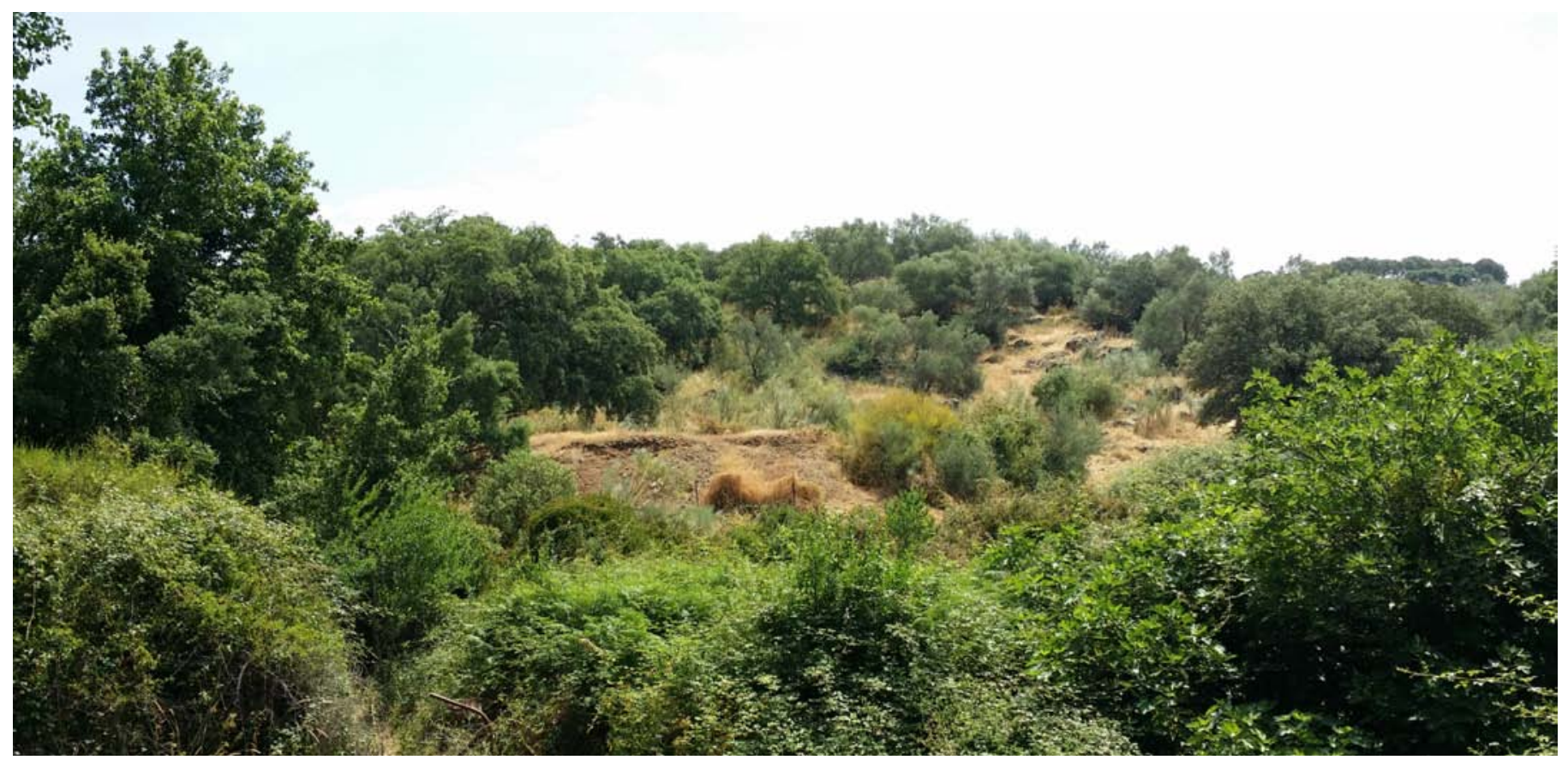

Figura 10. Vista general de las escombreras y antigua mina "Las Hormigas", Almonaster La Real.

Figure 10. General view of the dumps and old mine "Las Hormigas", Almonaster La Real.

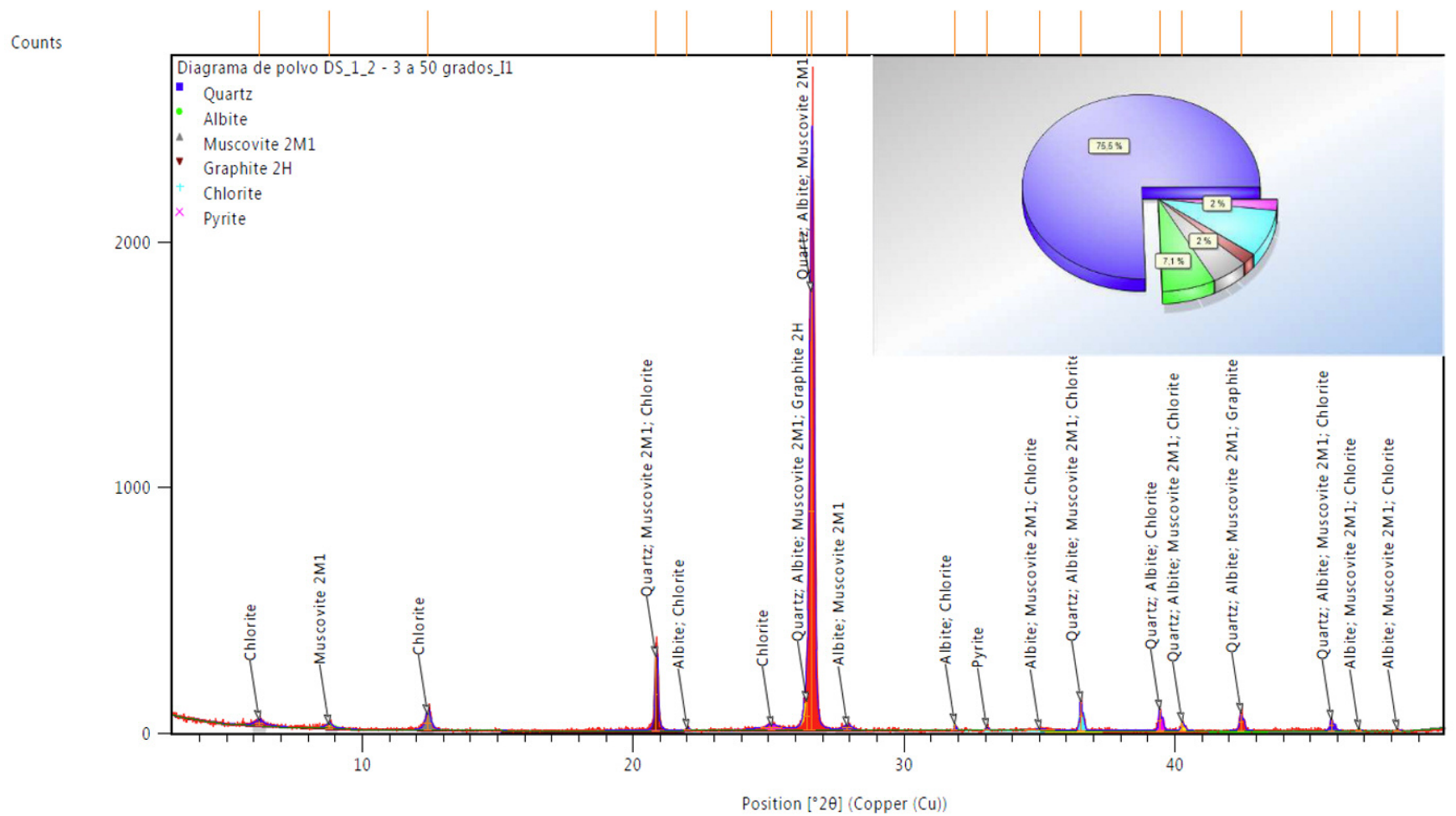

Figura 11. Resultados de difracción de rayos X realizada en una muestra de la mina "Las Hormigas". Nota: los porcentajes en el grafico se calculan de forma automática y tienen poca precisión.

Figure 11. X-ray diffraction performed on a sample from "Las Hormigas" mine. Note: the percentages in the graph are calculated automatically and with poor resolution. 
conjunto de datos obtenidos en esta campaña de exploración.

En la zona de la antigua labor minera de "San Carlos" se tomaron 5 muestras (Tabla 5), tanto de la entrada a la antigua labor como de las escombreras. Los análisis de TOC indican porcentajes atribuibles a grafito variables entre el 2 y el $9.6 \%$. En esta zona, la impresión que se obtiene tras la visita en campo es que la zona está fuertemente deformada tectónicamente, no parece haber una continuidad clara de las capas mineralizadas y el conjunto presenta buzamientos altos hacia el N. En cualquier caso los afloramientos son escasos y de mala calidad.

En el entorno de la antigua mina de "La Niña" (Fig. 9) se realizó una cartografía geológica de detalle y se tomaron muestras de grafito en sucesivas campañas (Fig. 12). Desde el arroyo de Alcalaboza y hacia el oeste se identifican dos zonas, una en la que la mineralización de grafito se puede observar de forma patente y masiva en superficie, y que es la que se

\begin{tabular}{|l|l|l|l|l|l|l|}
\hline \multicolumn{1}{|c|}{ MUESTRA } & \multicolumn{1}{|c|}{ FECHA } & \multicolumn{1}{c|}{ \% VOLÁTILES } & \multicolumn{1}{c|}{ \% C FIJO } & \multicolumn{1}{c|}{ \% CENIZAS } & \multicolumn{1}{c|}{ \% AZUFRE HUMEDAD } \\
\hline SC-1 & $14 / 07 / 2016$ & 1.45 & 6.73 & 91.82 & 0.0 & 0.77 \\
\hline SC-2 & $14 / 07 / 2016$ & 8.55 & 6.20 & 85.25 & 0.0 & 2.36 \\
\hline SC-3 & $14 / 07 / 2016$ & 6.70 & 1.94 & 91.37 & 0.014 & 3.45 \\
\hline SC-4 & $14 / 07 / 2016$ & 2.00 & 9.67 & 88.32 & 0.011 & 0.86 \\
\hline SC-5 & $14 / 07 / 2016$ & 6.73 & 0.00 & 93.92 & 0.0 & 0.75 \\
\hline
\end{tabular}

Tabla 5. Resultados de los análisis de las muestras obtenidas de la mina "San Carlos".

Table 5. Analytical results from the samples of the "San Carlos" mine.

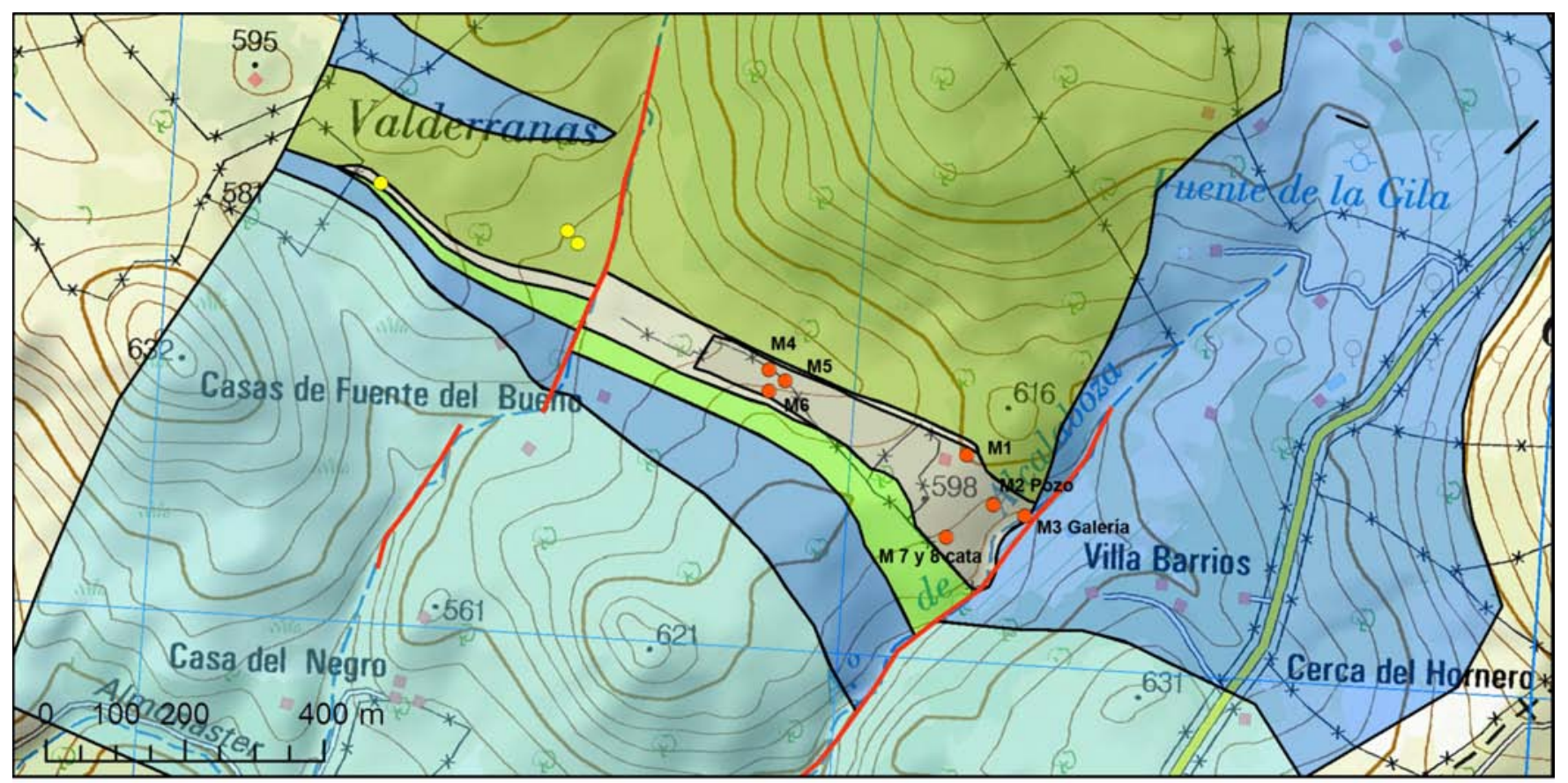

\section{Geología}

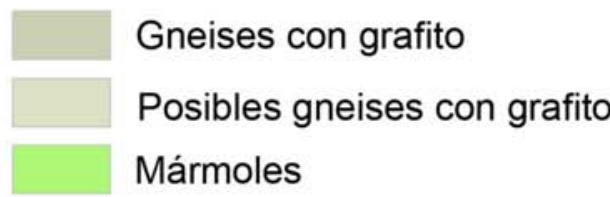

Intrusiones dioríticas

Gneises

Ortoanfibolitas toleiticas

Figura 12. Mapa geológico de detalle de la zona de La Niña.

Figure 12. Geological map of the La Niña area. 
muestrea, y otra inmediatamente adosada y hacia el oeste que se interpreta que es posible que la mineralización continúe. Esta última zona se interpreta en cartografía a partir de fotogeología mediante marcadores geomorfológicos del relieve, pero no se tiene constancia por dato/afloramiento alguno, tanto de subsuelo como de superficie. En la cartografía se observa que la mineralización se restringe a un área muy limitada entre dos arroyos, probablemente delimitada por fallas. En superficie se observa que la serie en general presenta buzamientos altos de entre 70-75 en el entorno de la antigua mina e incrementándose hasta su verticalización hacia el oeste, tal como insinúa la forma cartográfica resultante. Se observan evidencias notables de pliegues menores, sigmoides de cizalla y un profuso patrón de fracturación. A pesar de esta importante deformación interna el cuerpo mineralizado sí parece (al menos lo que se ve en superficie) estratoligado y estratiforme.

En total se tomaron 8 muestras en la zona (ver Fig. 12 y Tabla 6). Los porcentajes de grafito resultante oscilan entre el $4.8 \%$ y el $10.9 \%$, los valores más habituales son los que están entre el 5 y $6 \%$. Es de destacar por lo positivo que los porcentajes de azufre que contienen las muestras tomadas son mínimos (con un $0.056 \%$ de máxima). La ley media obtenida es de $5.65 \%$ de grafito. Entendemos que como las muestras están tomadas de superficie deben estar un poco lavadas y en profundidad la ley de grafito debería ser algo mayor. Por tanto, el valor de $5.65 \%$ de grafito en la mina de "La Niña" se puede tomar como un valor conservador.

En resumen, para el conjunto de zonas estudiadas en la Banda Metamórfica de Aracena los resultados de las analíticas indican contenidos máximos en Carbono fijo del $13 \%$ siendo lo habitual mucho menor, en torno al 5-6\%, con muestras frecuentes con contenidos incluso inferiores, todos ellos resultados similares a los obtenidos por el Lasheras et al. (1995).

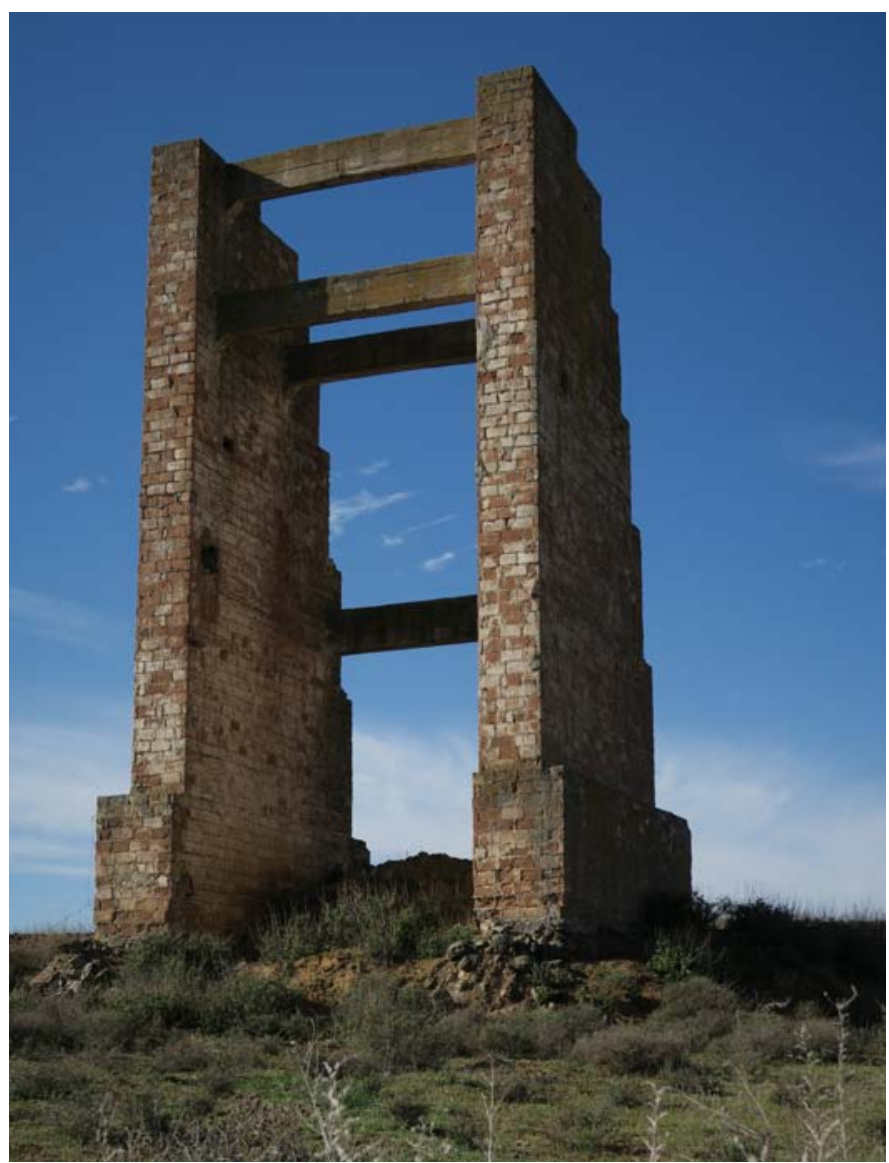

Figura 13. Aspecto actual del castillete de la mina de interior de Guadamur, cerrada en 1963.

Figure 13. Current aspect of the headframe of the Guadamur interior mine, closed in 1963.

\section{Zona de Guadamur}

Las antiguas explotaciones de grafito en el entorno de Guadamur, en explotación desde 1946 a 1963 por la sociedad Comercial Químico-Metalúrgica, se des-

\begin{tabular}{|l|l|l|l|l|l|l|}
\hline \multicolumn{1}{|c|}{ MUESTRA } & \multicolumn{1}{|c|}{ FECHA } & \multicolumn{1}{c|}{ \% VOLÁTILES } & \multicolumn{1}{c|}{ \% C FIJO } & \multicolumn{1}{c|}{ \% CENIZAS } & \multicolumn{1}{c|}{ \% AZUFRE HUMEDAD } \\
\hline LN-1 & $14 / 07 / 2016$ & 1.05 & 6.02 & 92.93 & 0.007 & 0.22 \\
\hline LN-2 & $14 / 07 / 2016$ & 0.86 & 5.28 & 93.86 & 0.0 & 0.21 \\
\hline LN-3 & $14 / 07 / 2016$ & 1.09 & 6.39 & 92.51 & 0.021 & 0.34 \\
\hline LN-4 & $03 / 11 / 2016$ & 1.71 & 4.88 & 93.41 & 0.017 & 0.47 \\
\hline LN-5 & $03 / 11 / 2016$ & 1.32 & 5.36 & 93.31 & 0.004 & 0.22 \\
\hline LN-6 & $03 / 11 / 2016$ & 2.06 & 5.21 & 92.72 & 0.016 & 0.42 \\
\hline LN-7 & $03 / 11 / 2016$ & 1.66 & 6.44 & 91.90 & 0.041 & 0.44 \\
\hline LN-8 & $03 / 11 / 2016$ & 5.23 & 10.92 & 83.34 & 0.056 & 0.45 \\
\hline
\end{tabular}

Tabla 6. Resultados de los análisis de las muestras obtenidas en el entorno de la mina "La Niña"

Table 6. Analytical results from the samples of the "La Niña" mine. 
arrollaron en torno a un filón (o zona de fractura muy vertical), encajado en metasedimentos precámbricos y tonalitas tardicinemáticas Variscas (Lasheras et al., 1995). Este filón, de varios centenares de metros de longitud fue explotado hasta unos 100 metros de profundidad mediante minería subterránea (Fig. 13) y presentaba una potencia estimada de dos-tres metros. Las leyes de esta mineralización oscilan entre el 3 y el $20 \%$ de grafito presentando alto grado de cristalización (Martín Méndez et al., 2015, 2017 y 2018). Sin embargo, estudios previos (Lasheras et al., $1995)$ indican valores más modestos (10\% para el mineral explotado y $2 \%$ en escombrera). Esta explotación llegó a conseguir concentrados de hasta el 80 $\%$ de grafito. También se observan en el entorno evidencias de grafito diseminado en los metasedimentos del Precámbrico y en pequeños filones de apenas $10-20 \mathrm{~cm}$ de potencia y longitudes cercanas al metro.

\section{Zona de Riaza-Sierra de Ayllón}

El grafito se presenta hacia el techo de algunas secuencias granodecrecientes en la serie pizarrosa negra del Silúrico (Fig. 14). Se encuentra acumulado en niveles de pocos centímetros en las zonas más finas, estas son relativamente abundantes y se identifican yacimientos en varias localidades (EI Muyo, Becerril, Madriguera y Serracín; Puche Riart and Pedrazuela González, 1988; Lasheras et al., 1995). A veces las zonas mineralizadas se encuentran en zonas de fractura o cizallas entre capas, evidenciando que el carbono está removilizado de la propia serie pizarrosa del Silúrico, probablemente durante el metamorfismo. Las analíticas realizadas dan contenidos en Carbono fijo relativamente bajos, siendo los de Becerril los más altos, concretamente una muestra tomada en la escombrera de la antigua mina de Becerril dio como resultado un contenido del $24,51 \%$ de carbono fijo atribuible al grafito, en línea con lo publicado por el IGME (Lasheras et al., 1995).

\section{Zona de Orea-Alto Tajo}

El grafito se encuentra asociado claramente a zonas de falla o cizalla que cortan las series pizarrosas oscuras del Ordovícico y Silúrico (ver Fig. 15). Los vesti-

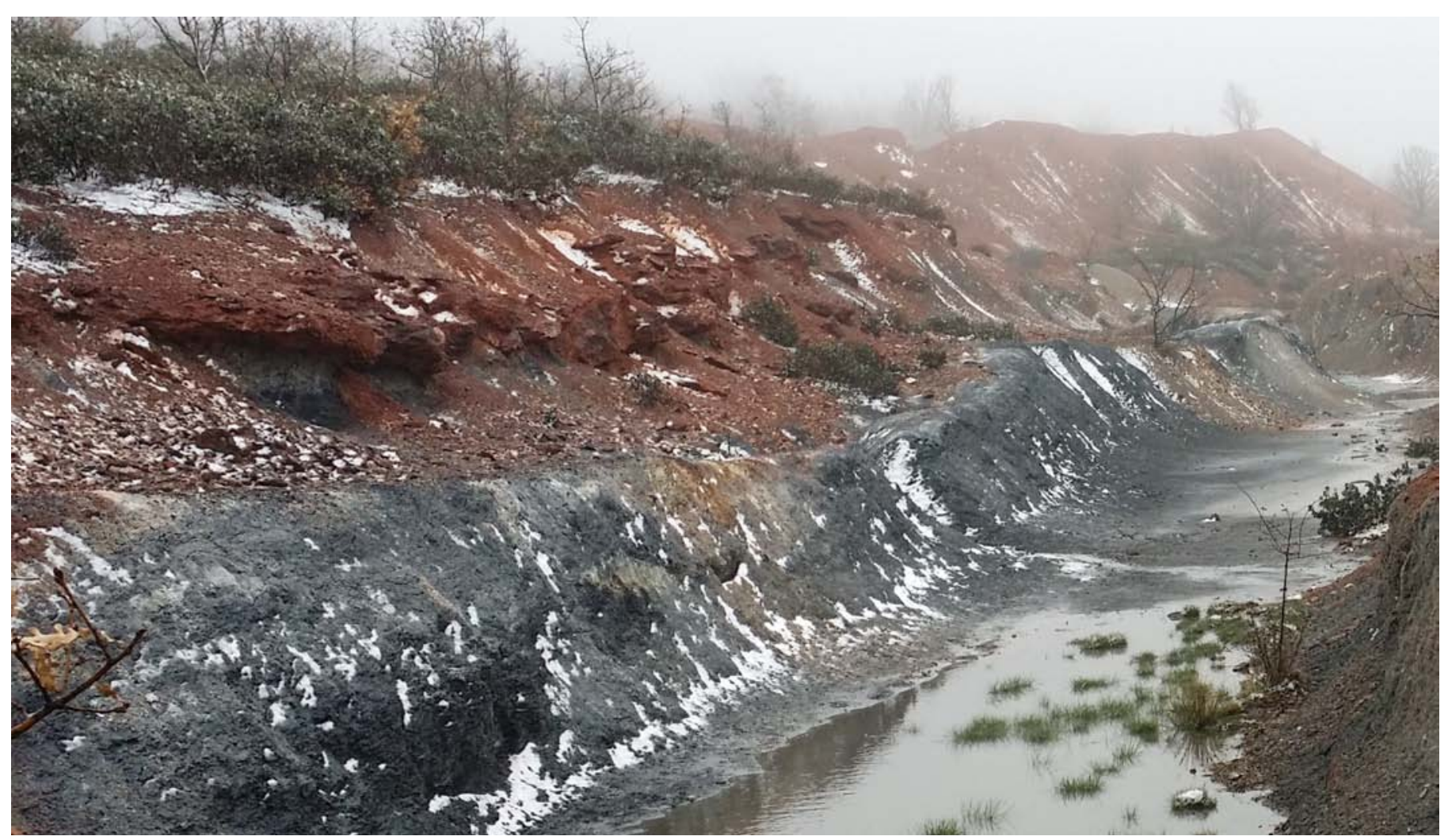

Figura 14. Aspecto de la serie pizarrosa negra del Silúrico que contiene la mineralización de grafito.

Figure 14. Silurian black shales containing the graphite mineralization. 


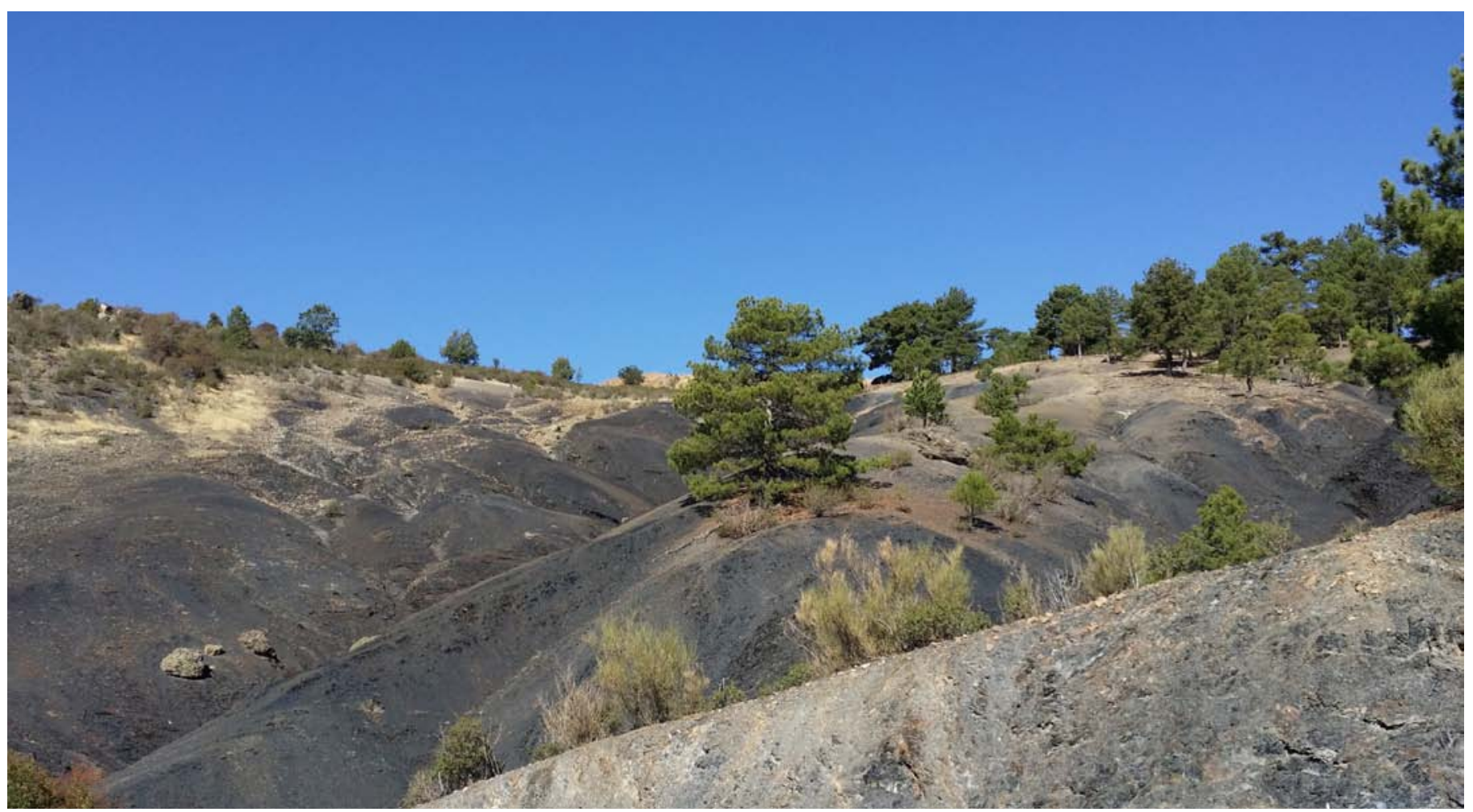

Figura 15. Aspecto de las series negras del Ordovícico y Silúrico en las que se encuentran los índicos de grafito. Figure 15. Ordovician and Silurian black shales with evidence of graphite.

gios de grafito son discontinuos y poco extensos, apenas unas decenas de metros. Los análisis dan consistentemente contenidos de Carbono fijo muy bajos, en torno al $1 \%$.

\section{Conclusiones}

Del resumen realizado de la situación actual a nivel internacional del grafito y a través de los datos y expectativas recopiladas se extrae que los precios del grafito van a tender al alza, es posible que de forma moderada en el corto plazo a la par que se incrementa la movilidad eléctrica. El incremento de la demanda es muy posible que se cubra tanto con los proyectos existentes como con los frutos positivos de las importantes campañas de exploración de grafito que se están llevando a cabo tanto en Canadá como en el sureste Africano y Asiático. En cualquier caso parece claro que tanto la demanda como la oferta se están incrementando de forma paulatina y las expectativas de futuro de este metal, al igual que el resto de componentes utilizados en la fabricación de baterías (cobalto o litio; ver Industrial Minerals and Chinese Customs 2018 o Roskill 2018a), son buenas. Además, desde el punto de vista de la trasformación se está tratando de fabricar grafito esférico en otros países, para no depender tanto de la producción de China (Roskill 2018a).

En cuanto a los trabajos de exploración realizados en España, indicar que las zonas objetivo presentan un interés exploratorio modesto, en general condicionado tanto por la escasez del recurso como por cuestiones medioambientales. Por ejemplo, es el caso de la zona con baja mineralización en Orea-Alto Tajo que se localiza en su mayor parte dentro de un Parque Natural.

Del conjunto de zonas estudiadas en la Banda Metamórfica de Aracena, las zonas de Las Hormigas, Aroche y San Carlos también presentan un bajo interés ya sea por falta de recurso o por cuestiones medioambientales (La antigua mina de Las Hormigas está dentro de varias figuras de protección ambiental).

La zona de la antigua mina de "La Niña" en la Banda Metamórfica de Aracena es la más prometedora ya que tiene una cierta continuidad cartográfica lo largo de más de un centenar de metros y presenta valores de Carbono fijo en torno al 5-6\% con picos cerca del $10 \%$. En esta zona no es posible hacer una prognosis fiable del subsuelo dada la complejidad estructural y litológica existente, por tanto cualquier 
continuación de la exploración pasa por la realización de sondeos y prospección geofísica.

\section{Agradecimientos}

Los autores agradecen a la Dirección General del Grupo SAMCA las facilidades dadas para la publicación de este trabajo.

\section{Referencias}

Brown, T.J., Idoine, N.E., Raycraft, E.R., Shaw, R.A., Hobbs, S.F., Everett, P., Deady, E.A. and Bide, T. 2018. World Mineral Production 2012-2016. Report of the British Geological Survey, Keyworth, Nottingham, $96 \mathrm{p}$.

Crespo, E., Luque, F.J., Fernández Rodríguez, C., Rodas, M., Díaz Azpiroz, M., Fernández Caliani, J.C. and Fernández Barrenechea, J.M. 2004. Significance of graphite occurrences in the Aracena Metamorphic Belt, Iberian Massif. Geological Magazine, 141 (6), 687-697.

Fernández Rodríguez, C., Fernández Caliani, J.C., Miras, A., Barrenechea, J.F., Luque, F.J. and Rodas, M. 1996. Nuevos datos geológicos sobre las mineralizaciones de grafito de la Banda Metamórfica de Aracena, Huelva (Macizo Ibérico Meridional). Geogaceta 20 (7), 1576-1577.

Ghilotti, G. 2018. Graphite industry prepares to spread risk beyond China. A Industrial Minerals report, $2 \mathrm{p}$.

Lasheras, A., Lombardero Barceló, M. and Rodriguez Suarez, J.I. 1995. Exploración de Grafito en España. Informe del Instituto Geológico y Minero de España, Madrid, $123 \mathrm{p}$.

Indian Bureau of Mines 2015. Indian Minerals Yearbook 2013, Graphite. 52 edition, 11 p.

Industrial Minerals Graphite Maps 2017. Industrial Minerals Graphite Maps, 1p.

Industrial Minerals and Chinese Customs 2018. Industrial Minerals and Chinese Customs Report, 3p.

Industrial Minerals 2010, Graphite prices rising daily. Industrial Minerals, June 29, accessed September 15, at http://www.indmin.com/Article/2620429/Graphite-pricesrising-daily.html.

Investing News Report 2018. Investing in Graphite. INR Dig Media Inc. Report, 18 p.

Lobato, E. 2009. Brasil Perfil da Grafita. J. Mendo Consultoría, informe inédito, $33 \mathrm{p}$.

Luque, F.J., Barrenechea, J.F. and Rodas, M. 1993. Graphite geothermometry in low and high temperature regimes: two case studies. Geological Magazine, 130, 501-511.

Martín Méndez, I., Boixereu, E. and Villaseca, C. 2015. Mineralogical and isotopic character of graphite deposits from the Anatectic Complex of Toledo, central Spain. Mineralium Deposita, 51, 575-590.

Martín-Méndez, I., Boixereu, E. and Villaseca, C. 2017. Caracterización mineralógica de los yacimientos de grafito del Complejo Anatéctico de Toledo. Macla, 21, 65-67.

Martín Mendez, I., Boixereu, E. and Villaseca, C. 2018. El grafito de Toledo ¿Un material para el futuro?. Tierra $y$ Tecnología, 51.

Mitchell, C.J. 1993. Industrial Minerals Laboratory Manual; Flake Graphite. Tehcnical Report WG/92/30 of the British Geological Survey. Keyworth, Nottingham, United Kingdom, 35 p.

Puche Riart, O. and Pedrazuela González, F. 1988. Estudio geológico de los yacimientos de grafito de la zona de Becerril (Segovia). Libro de abstracts del VII Congreso Internacional de Minería y Metalurgia, 16-22 Octubre 1988, Oviedo, 19 p.

Quesada, C., Fonseca, P.E., Munha, J., Oliveira, J.T. and Ribeiro, A. 1994. The Beja-Acebuches Ophiolite (Southern Iberia Variscan fold belt): geological characterization and geodynamic significance. Boletín Geológico y Minero de España, 105, 3-49.

Reichl, C., Schatz, M. and Zsak, G. 2018. World Mining Data, Mineral Production. Report of the Austrian Federal Ministry of Sustainability and Tourism, vol 33, 263 p.

Robinson, G.R., Hammarstrom, J.M., and Olson, D.W., 2017, Graphite. In: Schulz, K.J., DeYoung, J.H. Jr., Seal, R.R. II, and Bradley, D.C., (eds.), Critical mineral resources of the United States-Economic and environmental geology and prospects for future supply: U.S. Geological Survey Professional Paper 1802, p. J1-J24.

Rodas, M., Luque del Villar, F.J., Fernández Barrenechea, J.M., Fernández Caliani, J.C., Miras Ruiz, A. and Fernández Rodríguez, C. 2000. Graphite occurrences in the low-pressure/high-temperature metamorphic belt of the Sierra de Aracena (southern Iberian Massif). Mineralogical Magazine, 64 (5), 801-814.

Roskill 2018a. Battery raw material market tracker. In: Lithium, Cobalt, Graphite, Nickel and Manganese, 13 p.

Roskill 2018b. Global Industry, Markets \& Outlook. Newsletter report.

Roskill 2018c. Graphite on the up in 2018 as demand and prices rise. Natural and Synthetic Graphite.

USGS 2017. U.S. Graphite. Geological Survey, Mineral Commodity Summaries, January 2017, 2p.

Recibido: diciembre 2017

Revisado: febrero 2018

Aceptado: junio 2018

Publicado: marzo 2019 